\title{
Modeling and Dynamic Properties of a Four-Parameter Zener Model Vibration Isolator
}

\author{
Wen-ku Shi, Chen Qian, Zhi-yong Chen, Yang Cao, and Heng-hai Zhang
}

State Key Laboratory of Automotive Simulation and Control, Jilin University, Changchun 130022, China

Correspondence should be addressed to Zhi-yong Chen; chen_zy@jlu.edu.cn

Received 6 July 2016; Revised 22 October 2016; Accepted 3 November 2016

Academic Editor: Nerio Tullini

Copyright (C) 2016 Wen-ku Shi et al. This is an open access article distributed under the Creative Commons Attribution License, which permits unrestricted use, distribution, and reproduction in any medium, provided the original work is properly cited.

To install high-performance isolators in a limited installation space, a novel passive isolator based on the four-parameter Zener model is proposed. The proposed isolator consists of three major parts, namely, connecting structure, sealing construction, and upper and lower cavities, all of which are enclosed by four segments of metal bellows with the same diameter. The equivalent stiffness and damping model of the isolator are derived from the dynamic stiffness of the isolation system. Experiments are conducted, and the experiment error is analyzed. Test results verify the validity of the model. Theoretical analysis and numerical simulation reveal that the stiffness and damping of the isolator have multiple properties with different exciting amplitudes and structural parameters. In consideration of the design of the structural parameter, the effects of exciting amplitude, damp channel diameter, equivalent cylinder diameter of cavities, sum of the stiffness of the bellows at the end of the isolator, and length of damp channel on the dynamic properties of the isolator are discussed comprehensively. A design method based on the parameter sensitivity of the isolator's design parameter is proposed. Thus, the novel isolator can be practically applied to engineering and provide a significant contribution in the field.

\section{Introduction}

Microvibration usually refers to low-level mechanical vibration or disturbance in the microgravity environment; such vibrations can be induced by onboard mechanical moving systems, including mechanically tunable optical filters, cryocoolers, solar array drive mechanisms, antenna pointing mechanisms, and reaction wheel assemblies [1-3]. The main microvibration of onboard satellites focuses on the harmonic disturbances with a frequency range of $0.1-300 \mathrm{~Hz}[4,5]$. Microvibration in these satellites can take a long time to attenuate because environment damping in aerospace is minute [6]. This scenario can cause serious degradation, for example, downgrading of the image quality or positional accuracy of optical payloads, in the performance of high-precision payloads. Vibration isolators are often applied in vibration propagation paths to protect high-precision payloads from the effect of microvibration. Vibration isolators are designed for space application, such as advanced X-ray Astro-Physics Facility [7], James Webb Space Telescope [8], and satellite ultraquiet isolation technology experiment [9].
Passive isolation techniques are generally deployed in aerospace engineering, thereby providing high-performance and stability and requiring no external power [10-13]. With regard to passive isolators, the vibrations are dissipated through passive damping and the displacement transmissibility is low at a high frequency band [10, 12]. In general, passive isolators contain a high-performance damper, such as viscous fluid damper or viscoelastic composite damper. Traditional passive isolators such as metal coil spring and rubber are generally soft for vibration isolation at relatively low frequencies, which is not expected in practice [14]. Nonlinear stiffness and nonlinear damping have been recently reported to be beneficial to vibration isolation for low and high frequencies [15-17]. For microvibration isolation, liquid damping is generally applied, in which no dead zone of friction exists, thereby benefiting small amplitude vibration isolation [18]. A typical liquid damping isolator is called D-strut [12]; the critical part of this isolator is the passive damper. $\mathrm{D}$-strut is a three-parameter configuration instead of a conventional two-parameter configuration, which is called the Zener model [19]. This model, which greatly improves isolation 
performance, is composed of a parallel combination of a spring and an elastically supported damper.

To install high-performance isolators in a limited installation space, this paper presents a novel passive isolator with compact structures based on a four-parameter Zener model. The isolator is made using bellows and viscous fluid. In the isolator, the bellows act as a seal structure and provide supporting stiffness at the same time, and the damping structure can be arranged in the bellows conveniently, thereby ensuring that the size of the isolator is small enough for the isolator to be installed in a limited space. The dynamic characters of the isolator are influenced by the mass of flowing fluid pumped through the damp channel by vibratory displacements in the isolator, which introduces a fourth parameter for the conventional Zener model. The novel isolator shows some new characteristics in its equivalent stiffness and damping because of the additional parameter. These characteristics enhance the performance of the novel isolator in vibration isolation. The stiffness and damping properties of the novel isolator are investigated comprehensively in this study to determine how the fourth parameter influences the performance of the novel isolator. The equivalent stiffness and damping model of the isolator are obtained theoretically and tested numerically in Section 2. The experiments on stiffness and damping of the novel isolator under different excitation amplitudes are presented in Section 3. The effects of the designed parameters on the stiffness and damping of the novel isolator are analyzed in Section 4. To inspect the performance of the isolator comprehensively, the displacement transmission rate characteristics of the isolator are studied in Section 5. Finally, the summary and concluding remarks are presented in Section 6.

\section{Analysis on Stiffness and Damping of the Vibration Isolator}

As shown in Figure 1, the vibration isolator consists of three major parts, connecting structure, sealing construction, and upper and lower cavities, all of which are enclosed by four segments of metal bellows with the same diameter. The two cavities are filled with liquid, and a damp channel exists in the intermediate connecting plate, which connects the two cavities. The stiffness of the vibration isolator is provided when the bellows stretch and compress. The pressure in the two cavities changes while load $M$ vibrates up and down. The change in the two cavities causes a pressure difference between these cavities and forces liquid to flow through the two cavities via the damp channel. Energy loss occurs because of linear damping caused by the viscosity of the liquid and the quadratic damping caused by the eddy current, which occurs as a result of the sudden change in diameter of the liquid flow channel at the inlet and outlet. The quadratic damping performs well in suppressing resonance while having no negative effects on vibration isolation at high frequencies [20]. This energy loss results in the damping of the vibration isolator. In addition, the inertia of the liquid in the damp channel affects the stiffness and damping characteristics of the isolator, because the diameter of the damp channel is

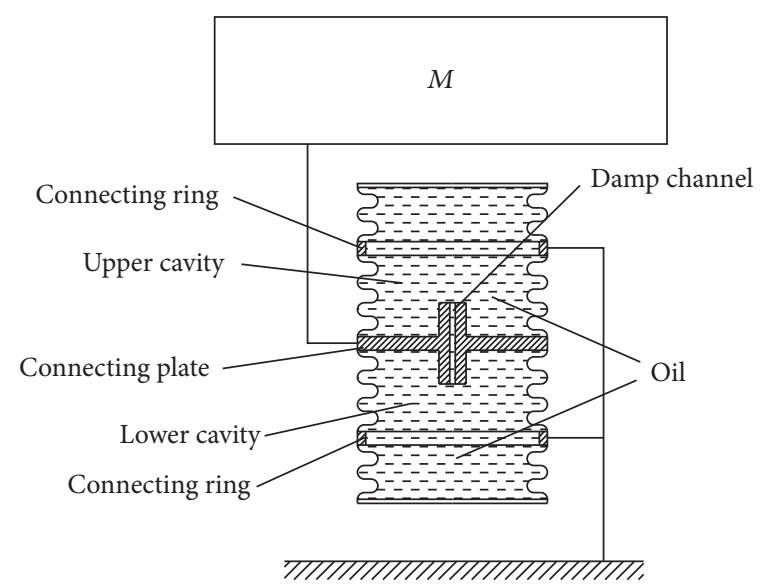

FIGURE 1: Structural diagram of the novel isolator with payload.

smaller than the equivalent diameter of the cavities, thereby increasing the liquid motion in the damp channel.

For a vibration isolation system, three or more vibration isolators are mounted. To achieve satisfactory performance of the vibration isolation system, reasonably matching the stiffness and damping properties of each vibration isolator is of importance. Therefore, this study establishes the relationship model between the structural parameters of the isolator and its stiffness and damping properties, comprehensively analyzes the stiffness and damping properties of the vibration isolator, and conducts a parameter sensitivity analysis on each key structural parameter of the isolator. This study lays the foundation for selecting the design parameters of the vibration isolator and reasonably matching the stiffness and damping properties of each vibration isolator.

2.1. Establishment of the Vibration Isolator Model. The working schematic diagram of the vibration isolator is shown in Figure 2(a). For the bellows, the longitudinal stiffness is recorded as $k_{a}$. The equivalent stiffness of the expansion and compression of liquid and radial of the bellows is recorded as $k_{b}$. When $k_{b}$ is three to five times larger than $k_{a}$, the effect of $k_{b}$ can be ignored in a certain range within the center of the firstorder support frequency [21]. The influence of $k_{b}$ is ignored when modeling in the case where the longitudinal stiffness of the bellows at both ends is not very different from that of the bellows at the middle. The stiffness of the middle bellows $k_{1}$ is parallel with fluid damping and with the equivalent liquid mass in the damp channel, whereas the stiffness of the bellows at both ends $k_{2}$ is in series with them. Linear damping $c_{l}$ and square damping $c_{q}$ occur when the liquid flows through the damp channel. The axial deformation response of the upper and lower cavities of the vibration isolator is $x_{2}$, which is the displacement response of the equivalent liquid mass in the damp channel. The vibration isolator can be equivalent to the model with two degrees of freedom, as shown in Figure 2(b), in which $m_{\mathrm{eq}}$ acts as the fourth parameter of the conventional Zener model. The significance of each parameter is as follows:

$A_{\text {eq }}$ : equivalent cross-sectional area of the bellows. $k_{2}$ : sum of the stiffness of bellows at both ends. 


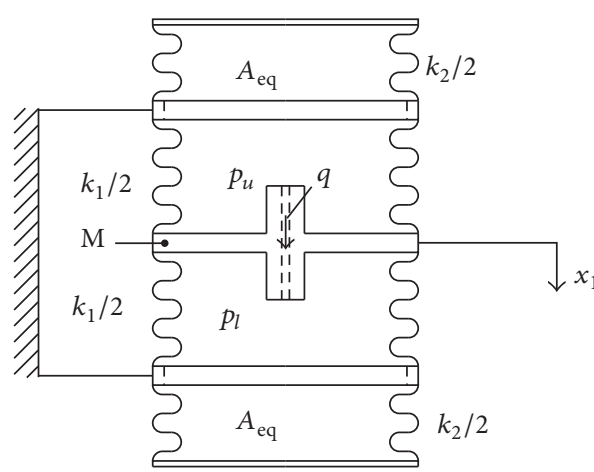

(a)

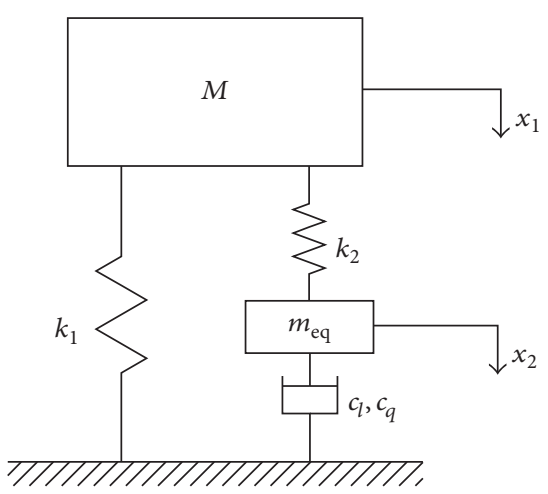

(b)

FIGURE 2: (a) Working schematic diagram of the isolator. (b) Equivalent system with two degrees of freedom.

$k_{1}$ : sum of the stiffness of the middle bellows.

$M$ : mass of the load.

$p_{u}$ : pressure change of the upper cavity.

$p_{l}$ : pressure change of the lower cavity.

$q$ : flow of fluid in the damp channel.

$x_{1}$ : displacement of excitation.

$x_{2}$ : axial deformation response of the upper and lower cavities.

$m_{\mathrm{eq}}$ : equivalent liquid mass flow through the damp channel.

$c_{l}$ : linear damping coefficient of damp channel.

$c_{q}$ : square damping coefficient of damp channel.

The equivalent cylindrical cross-sectional area of the bellows can be calculated as follows [22]:

$$
A_{\mathrm{eq}}=\frac{\pi\left(D_{b}^{2}+d_{b}^{2}\right)}{8} .
$$

In the formula, $D_{b}$ is the large diameter of the bellows, whereas $d_{b}$ is the small diameter of the bellows.

The flow of liquid $Q$ between the two cavities of the isolator can be expressed as

$$
Q=A_{\mathrm{eq}} \dot{x}_{2}
$$

The change of pressure in the two cavities shown in Figure 2(a) is

$$
\begin{aligned}
\Delta p_{u} & =\frac{-k_{2}\left(x_{1}-x_{2}\right)}{2 A_{\mathrm{eq}}}, \\
\Delta p_{l} & =\frac{k_{2}\left(x_{1}-x_{2}\right)}{2 A_{\mathrm{eq}}} .
\end{aligned}
$$

When the fluid flows in the damp channel, the total pressure difference between the upper and lower cavities equals the sum of pressure loss caused by the damp channel and the equivalent pressure loss caused by the inertia of the liquid flowing in the damp channel; that is,

$$
\Delta p_{u}-\Delta p_{l}+I \cdot \dot{Q}(t)+\Delta P=0 .
$$

In the preceding formula (4), $I$ is the fluid inductance of the fluid in the damp channel. It can be expressed as follows:

$$
I=\frac{\rho l_{c}}{A_{c}}
$$

In formula (5), $A_{c}$ is the cross-sectional area of the damp channel and $l_{c}$ is the length of the damp channel.

$\Delta P$ is the sum of linear pressure loss $\Delta P_{1}$, inlet pressure loss $\Delta P_{2}$, and outlet pressure loss $\Delta P_{3}$ caused by the damp channel; that is,

$$
\Delta P=\Delta P_{1}+\Delta P_{2}+\Delta P_{3} .
$$

$\Delta P_{1}, \Delta P_{2}$, and $\Delta P_{3}$ are calculated as follows:

$$
\begin{aligned}
\Delta P_{1} & =\frac{128 \mu l_{c}}{\pi d_{c}^{4}} Q, \\
\Delta P_{2} & =\frac{1}{2} \rho \zeta_{1}\left(\frac{Q}{A_{c}}\right)^{2}, \\
\Delta P_{3} & =\frac{1}{2} \rho \zeta_{2}\left(\frac{Q}{A_{c}}\right)^{2} .
\end{aligned}
$$

In formula $((7 \mathrm{a}),(7 \mathrm{~b})$, and $(7 \mathrm{c})), d_{c}$ is the diameter of the damp channel; $\zeta_{1}$ and $\zeta_{2}$ are the sudden expansion and sudden contraction resistance coefficients caused by the sudden change in the cross-sectional area of the liquid flow channel at both ends of the damp channel, which are determined by Reynolds number (be related to $\omega$ and $X_{2}$ ) when the fluid flow through the damp channel. The damp channel can be found in the relevant manual.

The following can be obtained by arranging all the preceding formulas:

$$
\begin{aligned}
& \frac{\pi \rho l_{c} d_{\mathrm{eq}}^{4}}{4 d_{c}^{2}} \ddot{x}_{2}+\frac{8 \pi \mu l_{c} d_{\mathrm{eq}}^{4}}{d_{c}^{4}} \dot{x}_{2}+\frac{\pi \rho d_{\mathrm{eq}}^{6}\left(\zeta_{1}+\zeta_{2}\right)}{8 d_{c}^{4}} \dot{x}_{2}\left|\dot{x}_{2}\right| \\
& -k_{2}\left(x_{1}-x_{2}\right)=0 .
\end{aligned}
$$


In formula (8), $d_{\mathrm{eq}}$ is the equivalent cylindrical diameter of the bellows.

As for the equivalent mass of the liquid in the damp channel in Figure 2(b),

$$
m_{\mathrm{eq}} \ddot{x}_{2}+c_{1} \dot{x}_{2}+c_{q} \dot{x}_{2}\left|\dot{x}_{2}\right|-k_{2}\left(x_{1}-x_{2}\right)=0 .
$$

In formula (9), $c_{l}$ is the linear damping coefficient caused by the viscosity of the fluid, $c_{q}$ is the square damping coefficient of the eddy current caused by the sudden change of the cross-sectional area of the fluid flow channel and the inertia of the fluid, and $m_{\mathrm{eq}}$ is the equivalent mass of the fluid in the damp channel. The comparison of Formula (8) with Formula (9) shows that

$$
\begin{aligned}
c_{l} & =\frac{8 \pi \mu l_{c} d_{\mathrm{eq}}^{4}}{d_{c}^{4}}, \\
c_{q} & =\frac{\pi \rho d_{\mathrm{eq}}^{6}}{8 d_{c}^{4}}\left(\zeta_{1}+\zeta_{2}\right), \\
m_{\mathrm{eq}} & =\frac{\pi \rho l_{c} d_{\mathrm{eq}}^{4}}{4 d_{c}^{2}},
\end{aligned}
$$

where $m_{c}=\rho l_{c} A_{c}$ is the actual mass of the fluid in the damp channel. It is plugged into formula (11); thus, the following is determined:

$$
m_{\mathrm{eq}}=d_{\mathrm{eq}}^{4} d_{c}^{-4} m_{c}
$$

The cross-sectional area of the damp channel is usually smaller than the equivalent cross-sectional area of the cavities of the isolator. Thus, the inertia effect of the fluid in the damp channel is enhanced.

The pressure in the upper and lower cavity of the isolator cannot be transferred to the base (the connecting ring), so the reaction force can only via $k_{1}$ and $k_{2}$ be transferred to the base, while $k_{2}$ is distorted by a displacement $x_{1}-x_{2}$. Actually, $k_{2}$ is directly connected to the base, so Figure 2(b) cannot reflect the actual state of the isolator at this time. The reaction force transferred to the base can be obtained by Figure 2(a):

$$
F_{T}=k_{1} x_{1}+k_{2}\left(x_{1}-x_{2}\right) \text {. }
$$

If $x_{1}$ is harmonic excitation, then $x_{1}$ and $x_{2}$ can be expressed as follows:

$$
\begin{aligned}
& x_{1}=X_{1} e^{j \omega t}, \\
& x_{2}=X_{2} e^{j(\omega t+\theta)} .
\end{aligned}
$$

To simplify the research, the equivalent damping coefficient of Formula (9) is used as follows according to the principle of energy equivalence:

$$
\bar{c}_{p}=c_{l}+\frac{8 \omega X_{2}}{3 \pi} c_{q}
$$

where $\bar{c}_{p}$ is obtained by calculating the energy dissipated in the system by considering only the dominant harmonic as an approximation.
Formulas (14) and (15) are plugged into formula (9), and the following formula can be obtained:

$$
\begin{aligned}
& \frac{X_{2}}{X_{1}}=\frac{k_{2}}{\sqrt{\left(k_{2}-m_{\mathrm{eq}} \omega^{2}\right)^{2}+\left(\bar{c}_{p} \omega\right)^{2}}}, \\
& e^{j \theta}=\frac{k_{2}-m_{\mathrm{eq}} \omega^{2}-\bar{c}_{p} j \omega}{\sqrt{\left(k_{2}-m_{\mathrm{eq}} \omega^{2}\right)^{2}+\left(\bar{c}_{p} \omega\right)^{2}}} .
\end{aligned}
$$

According to formulas (13), (14), (16), and (17), the span dynamic stiffness of the vibration isolator is as follows:

$$
\begin{aligned}
k_{s}= & \frac{F_{T}}{x_{1}} \\
= & k_{1}+k_{2}-k_{2}^{2} \frac{k_{2}-m_{\mathrm{eq}} \omega^{2}}{\left(k_{2}-m_{\mathrm{eq}} \omega^{2}\right)^{2}+\left(\bar{c}_{p} \omega\right)^{2}} \\
& +\bar{c}_{p} \frac{k_{2}^{2} j \omega}{\left(k_{2}-m_{\mathrm{eq}} \omega^{2}\right)^{2}+\left(\bar{c}_{p} \omega\right)^{2}} .
\end{aligned}
$$

According to SAE (Society of Automotive Engineers) $1085 \mathrm{~B}$ standards, the real part of $k_{s}$ is in-phase dynamic stiffness, which denote the elastic part of the vibration isolator, whereas the imaginary part is orthogonal dynamic stiffness, which reflect the damping characteristics of the vibration isolator. Thus, the equivalent stiffness in Figure 2(b) is as follows:

$$
k_{\mathrm{eq}}=k_{1}+k_{2}-k_{2}^{2} \frac{k_{2}-m_{\mathrm{eq}} \omega^{2}}{\left(k_{2}-m_{\mathrm{eq}} \omega^{2}\right)^{2}+\left(\bar{c}_{p} \omega\right)^{2}} .
$$

The equivalent damping coefficient is

$$
c_{\mathrm{eq}}=\bar{c}_{p} \frac{k_{2}^{2}}{\left(k_{2}-m_{\mathrm{eq}} \omega^{2}\right)^{2}+\left(\bar{c}_{p} \omega\right)^{2}} .
$$

2.2. Analysis of the Equivalent Stiffness Characteristic. If $k_{0}=$ $\left.k_{\mathrm{eq}}\right|_{\omega \rightarrow 0}$ and $k_{\mathrm{\infty}}=\left.k_{\mathrm{eq}}\right|_{\omega \rightarrow 0}$, then, according to Formula (19), the following can be obtained:

$$
\begin{aligned}
k_{0} & =k_{1}, \\
k_{\infty} & =k_{1}+k_{2} .
\end{aligned}
$$

The numerical value of $k_{0}$ is equal to the sum of the stiffness of the bellows in the middle of the isolator, which is the quasistatic stiffness of the vibration isolator, and of the static support stiffness of the vibration isolator while the isolator is working. According to the demand of system stiffness, $k_{0}$ should be matched first. When $\omega \rightarrow 0$, the reaction force of the mounting base is $\left.F_{T}\right|_{\omega \rightarrow 0}=k x_{1}$. When combined with formula (13), we easily determine that $x_{1}=x_{2}$. The axial deformation of the two cavities of the isolator equals the displacement of the external excitation. The bellows at 
the end of the isolator do not result in axial deformation. When $\omega \rightarrow \infty$, the reaction force of the mounting base is $\left.F_{T}\right|_{\omega \rightarrow 0}=k x_{1}+k_{2} x_{1}$; thus, $x_{2}=0$. The two cavities of the vibration isolator do not result in axial deformation, and their volumes remain constant. At this time, the axial deformation of both bellows at the end of the vibration isolator equals the displacement of the external excitation. Thus, the fluid in the damp channel is no longer flowing, which results in dynamic hardening. This scenario indicates that the damp channel is equivalently blocked.

According to formula (9), the natural frequency of the fluid in the damp channel is as follows:

$$
\omega_{n}=\sqrt{\frac{k_{2}}{m_{\mathrm{eq}}}} .
$$

This natural frequency is plugged into formula (19). When $\omega=\omega_{n}$, the following is obtained:

$$
\left.k_{\mathrm{eq}}\right|_{\omega=\omega_{n}}=k_{\mathrm{\infty}}=k_{1}+k_{2} .
$$

Formula (11) is plugged into formula (22):

$$
\omega_{n}=\frac{d_{c}}{d_{\mathrm{eq}}^{2}} \sqrt{\frac{k_{2}}{\rho l_{c}}} .
$$

The combination of formulas (21), (23), and (24) determine that, for a vibration isolator with a specific parameter, the various curves of the equivalent stiffness of the isolator with the change of excitation frequency are always through two certain points $\left(0, k_{1}\right)$ and $\left(\omega_{n}, k_{1}+k_{2}\right)$, regardless of how the external excitation amplitude changes. When $\omega \rightarrow \infty$, $k_{\text {eq }} \rightarrow k_{1}+k_{2}$; thus, parameter identification can be made using the characteristics of equivalent stiffness [23].

If $\bar{c}_{p}$ is constant, then the derivative of $k_{\text {eq }}$ can be obtained with respect to $\omega$ in formula (19), and the two extreme points of $k_{\text {eq }}$, namely, $\omega_{v}$ and $\omega_{p}$, can be obtained:

$$
\begin{aligned}
\xi & =\frac{\bar{c}_{p}}{2 \sqrt{k_{2} m_{\mathrm{eq}}}}, \\
\omega_{v} & =\omega_{n} \sqrt{1-2 \xi} \quad \text { if } \xi<\frac{1}{2}, \\
\omega_{p} & =\omega_{n} \sqrt{1+2 \xi} .
\end{aligned}
$$

$\xi$ is the equivalent damping ratio of the vibration isolator, $\omega_{v}$ is the minimum point of $k_{\text {eq }}$, and $\omega_{p}$ is the maximum point of $k_{\text {eq }}$.

Formulas (25) to (27) are plugged into formula (19), and the value of the extreme point of $k_{\text {eq }}$ can be obtained:

$$
\begin{aligned}
& k_{v}=k_{1}+k_{2}-\frac{k_{2}}{4 \xi(1-\xi)} \quad \text { if } \xi<\frac{1}{2}, \\
& k_{p}=k_{1}+k_{2}+\frac{k_{2}}{4 \xi(1+\xi)},
\end{aligned}
$$

TABLE 1: Law by which $k_{\text {eq }}$ changes with $\omega$.

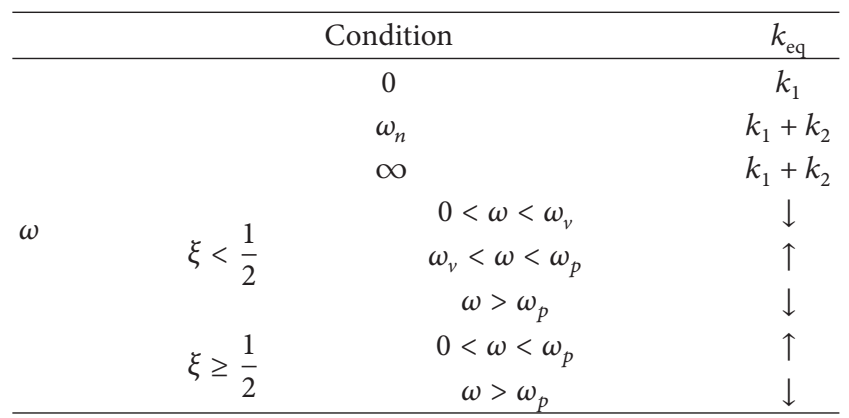

TABLE 2: Law by which $\xi$ affects the key points of the equivalent stiffness.

\begin{tabular}{cccccc}
\hline & Condition & $\omega_{v}$ & $k_{v}$ & $\omega_{p}$ & $k_{p}$ \\
\hline$\xi$ & $\downarrow$ & $\uparrow$ & $\uparrow$ & $\downarrow$ \\
$\xi$ & $\xi \geq \frac{1}{2}$ & - & - & $\uparrow$ & $\downarrow$ \\
\hline
\end{tabular}

where $\left.\omega_{v}\right|_{\xi=1 / 2}=0,\left.k_{v}\right|_{\xi=1 / 2}=k_{0}$. Therefore, when $\xi=$ $1 / 2$, points $\left(\omega_{v}, k_{v}\right)$ and $\left(0, k_{1}\right)$ coincide. When $\xi>1 / 2$, no minimum point exists.

$\omega_{v}<\omega_{n}<\omega_{p}$ can be obtained using formulas (26) and (27), whereas $k_{v}<k_{0}<k_{\infty}<k_{p}$ can be obtained using formulas (21), (28), and (29). Thus, the rule of change of $k_{\text {eq }}$ with excitation frequency $\omega$ is as follows: (1) $\xi<1 / 2$. When $\omega \in\left(0, \omega_{v}\right], k_{\text {eq }}$ begins to decrease from point $\left(0, k_{1}\right)$ with the increase of frequency, and the minimum value $k_{v}$ appears at $\omega_{v}$. With the increase of $\xi, \omega_{v}$ decreases while $k_{v}$ increases. When $\omega \in\left(\omega_{v}, \omega_{p}\right], k_{\mathrm{eq}}$ increases and goes through a certain point $\left(\omega_{n}, k_{1}+k_{2}\right)$ with the increase of frequency; the maximum value $k_{p}$ also appears at $\omega_{p}$. With the increase of $\xi$, $\omega_{p}$ decreases while $k_{p}$ increases. When $\omega>\omega_{p}, k_{\mathrm{eq}}$ decreases with the increase of frequency. When $\omega \rightarrow \infty$, $k_{\text {eq }} \rightarrow k_{1}+k_{2}$. (2) $\xi \geq 1 / 2$. When $\omega \in\left(0, \omega_{p}\right], k_{\text {eq }}$ increases and goes through a certain point $\left(\omega_{n}, k_{1}+k_{2}\right)$ with the increase of frequency. Moreover, the maximum value appears at $\omega_{p}$. With the increase of $\xi, \omega_{p}$ increases while $k_{p}$ decreases, and $\left.k_{p}\right|_{\xi \rightarrow \infty}=k_{1}+k_{2}$. When $\omega>\omega_{p}, k_{\text {eq }}$ decreases with the increase of frequency. When $\omega \rightarrow \infty, k_{\mathrm{eq}} \rightarrow k_{1}+k_{2}$. $\left.k_{p}\right|_{\xi \rightarrow \infty}=k_{1}+k_{2}$. Therefore, when the damping ratio of the system is sufficiently large, the maximum point $\left(\omega_{p}, k_{p}\right)$ disappears. In this case, $k_{\mathrm{eq}} \in\left(k_{1}, k_{1}+k_{2}\right)$.

According to the preceding analysis, the law by which $k_{\text {eq }}$ changes with $\omega$ can be summarized in Table 1.

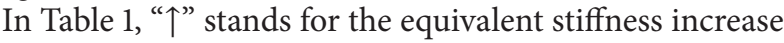
along with the increase of frequency, whereas “ $\downarrow$ ” stands for the equivalent stiffness decrease along with the increase of frequency.

The law by which $\xi$ affects the key points of the equivalent stiffness is shown in Table 2.

In Table 2, “ $\uparrow$ ” stands for the key point of equivalent stiffness increase along with the increase of $\xi$, “ $\downarrow$ ” stands for the key point of equivalent stiffness decrease along with the increase of $\xi$, and “-” means not existing. 
TABLE 3: Rule by which $c_{\text {eq }}$ changes with $\omega$.

\begin{tabular}{|c|c|c|c|}
\hline \multicolumn{3}{|c|}{ Condition } & $c_{\mathrm{eq}}$ \\
\hline \multirow{5}{*}{$\omega$} & \multicolumn{2}{|c|}{0} & $c_{l}$ \\
\hline & \multicolumn{2}{|c|}{$\infty$} & 0 \\
\hline & $\sqrt{2}$ & $0<\omega<\omega_{c}$ & $\uparrow$ \\
\hline & $5<\overline{2}$ & $\omega>\omega_{c}$ & $\downarrow$ \\
\hline & & & $\downarrow$ \\
\hline
\end{tabular}

2.3. Property Analysis of Equivalent Damping Coefficient. $c_{0}=$ $\left.c_{\text {eq }}\right|_{\omega \rightarrow 0}$ and $c_{\infty}=\left.c_{\text {eq }}\right|_{\omega \rightarrow 0}$ are assumed; thus, according to formulas (15) and (20):

$$
\begin{aligned}
c_{0} & =c_{l}, \\
c_{\infty} & =0 .
\end{aligned}
$$

For different external incentives, the equivalent damping of the isolator always goes through two certain points, namely, $\left(0, c_{l}\right)$ and $(\infty, 0)$. When $\omega \rightarrow 0$, the fluid in the damp channel flows slowly, and the damping of the vibration isolator mainly consists of linear damping. When $\omega \rightarrow \infty$, the dynamic hardening of the fluid in the damp channel occurs, the fluid in the damp channel no longer flows, and the damping coefficient of the isolator is zero. This finding is consistent with that of the previous analysis. The vibration isolator can satisfy the small damping of the vibration isolation system at high frequency.

The derivative of $c_{\mathrm{eq}}$ is obtained with respect to $\omega$ in formula (20), and the frequency of the extreme point of the equivalent damping coefficient is as follows:

$$
\omega_{c}=\omega_{n} \sqrt{1-2 \xi^{2}} \quad \text { if } \xi<\frac{\sqrt{2}}{2} .
$$

Formula (31) is plugged into formula (20), and the peak value of the equivalent damping coefficient can be obtained:

$$
c_{c}=\frac{k_{2}}{\omega_{n}} \frac{1}{2 \xi\left(1-\xi^{2}\right)} \quad \text { if } \xi<\frac{\sqrt{2}}{2} .
$$

$\left.\omega_{c}\right|_{\xi=\sqrt{2} / 2}=0,\left.c_{c}\right|_{\xi=\sqrt{2} / 2}=\sqrt{2} k_{2} / \omega_{n}=c_{l}$. Therefore, when $\xi=\sqrt{2} / 2$, the maximum value occurs at $\omega=0$, and when $\xi \geq \sqrt{2} / 2$, no extreme point exists.

Thus, the rule of change of $c_{\text {eq }}$ with excitation frequency $\omega$ is as follows: (1) $\xi<\sqrt{2} / 2$. $c_{\text {eq }}$ increases first and then decreases with the increase of $\omega$. The maximum value $c_{c}$ appears at $\omega_{c}$, and peak point $\omega_{c}$ decreases with the increase of $\xi$. When $\xi<\sqrt{3} / 3, c_{c}$ increases first and then decreases with the increase of $\xi$. When $\sqrt{3} / 3<\xi<\sqrt{2} / 2, c_{c}$ increases with the increase of $\xi$. (2) $\xi \geq \sqrt{2} / 2$. $c_{\text {eq }}$ decreases with the increase of $\omega$, and the maximum value is $c_{0}=c_{l}$.

According to the preceding formulas above, the rule by which $c_{\text {eq }}$ changes with $\omega$ can be summarized in Table 3 .

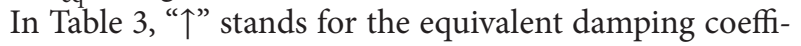
cient increase along with the increase of frequency, whereas “ $\downarrow$ " stands for the equivalent damping coefficient decrease along with the increase of frequency.
TABLE 4: Rule by which $\xi$ affects the key points of the equivalent damping coefficient.

\begin{tabular}{cccc}
\hline & Condition & $\omega_{c}$ & $c_{c}$ \\
\hline$\xi<\frac{\sqrt{3}}{3}$ & $\downarrow$ & $\downarrow$ \\
$\xi$ & $\frac{\sqrt{3}}{3}<\xi<\frac{\sqrt{2}}{2}$ & $\downarrow$ & $\uparrow$ \\
$\xi$ & $\geq \frac{\sqrt{2}}{2}$ & - & - \\
\hline
\end{tabular}

The rule by which $\xi$ affects the key points of the equivalent damping coefficient is shown in Table 4.

In Table 4, “ $\uparrow$ " stands for the key point of equivalent damping coefficient increase along with the increase of $\xi$, “ $\downarrow$ ” stands for the key point of equivalent damping coefficient decreases with the increase of $\xi$, and “-” means not existing.

2.4. Reliability Verification on the Model of Equivalent Stiffness and Equivalent Damping Ratio of the Vibration Isolator. Formulas (9) and (13) are changed into a dimensionless form to facilitate the numerical simulation. $\lambda=\omega / \omega_{n}, \tau=\omega_{n} t$, $\dot{x}_{1}(t)=\dot{x}_{1}(\tau) \omega_{n}, \dot{x}_{2}(t)=\dot{x}_{2}(\tau) \omega_{n}, x_{0}=0.06 \mathrm{~mm}, z_{1}=$ $x_{1}(\tau) / x_{0}, z_{2}=x_{2}(\tau) / x_{0}, \zeta_{l}=c_{l} \omega_{n} / k_{2}, \zeta_{q}=c_{q} x_{0} \omega_{n}^{2} / k_{2}$, $f_{0}=k_{2} x_{0}, f_{T}=F_{T} / f_{0}$, and $N=k_{1} / k_{2}$ are defined, and the equations of the dimensionless forms are as follows:

$$
\begin{aligned}
\ddot{z}_{2}+\zeta_{l} \dot{z}_{2}+\zeta_{q} \dot{z}_{2}\left|\dot{z}_{2}\right| & =z_{1}, \\
f_{T} & =(N+1) z_{1}-z_{2} .
\end{aligned}
$$

In formulas (33a) and (33b), $\lambda, z_{1}, z_{2}$, and $f_{T}$ are the dimensionless forms of $\omega, x_{1}, x_{2}$, and $F_{T}$, respectively. $k_{\text {eq }}$ and $c_{\mathrm{eq}}$ are identified on the basis of the equation $k_{s}=k_{\mathrm{eq}}+c_{\mathrm{eq}} j \omega$. The related parameters are $k_{2}=90.07 \mathrm{~N} / \mathrm{mm}, N=0.2$, $d_{\mathrm{eq}}=25 \mathrm{~mm}, d_{c}=2 \mathrm{~mm}$, and $\zeta_{l}=0.1172 . \zeta_{q}$ is calculated according to $\zeta_{1}$ and $\zeta_{2}$, which are queried from the simulation results in real time. The comparison of equivalent stiffness and damping coefficient results of the model with the results of the numerical simulation is shown in Figure 3.

The equivalent stiffness and damping coefficient results of the model and the numerical simulation match well; thus, the reliability of the model can be verified. However, a numerical simulation must be performed first to determine the parameter $\bar{c}_{p}$ of the model by obtaining the value of $X_{2}$. Thus, the following quantitative analysis is based on the numerical simulation.

2.5. Analysis of the Effect of the Fourth Parameter $m_{e q}$. The numerical simulations of the equivalent stiffness and the equivalent damping coefficient results of the isolator when $m_{\mathrm{eq}} \neq 0$ and $m_{\mathrm{eq}}=0$ are shown in Figures 4 and 5, respectively. These simulations clearly determine how the fourth parameter $m_{\mathrm{eq}}$ influences the characteristic of the novel isolator.

Figure 4 shows that when $m_{\mathrm{eq}}=0$, the valley point $\left(\omega_{v}, k_{v}\right)$ and the peak point $\left(\omega_{p}, k_{p}\right)$ disappear. Thus, the fourth parameter $m_{\mathrm{eq}}$ introduces a valley point $\left(\omega_{v}, k_{v}\right)$ and a peak point $\left(\omega_{p}, k_{p}\right)$ to the equivalent stiffness characteristic of the novel isolator. When $m_{\mathrm{eq}} \neq 0$, if $\zeta_{q}$ is given a large 


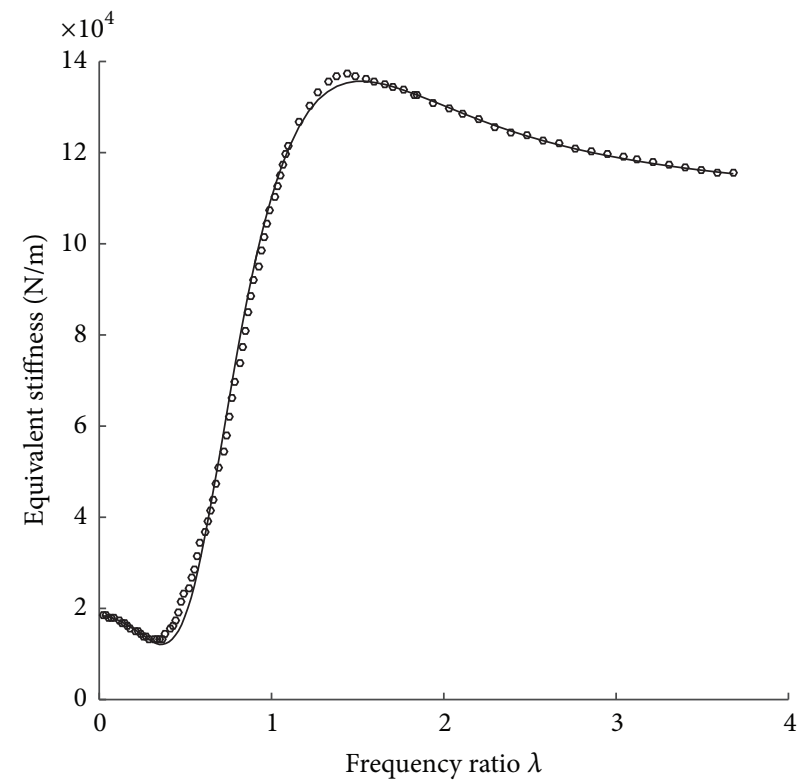

- Numerical

- Model

(a)

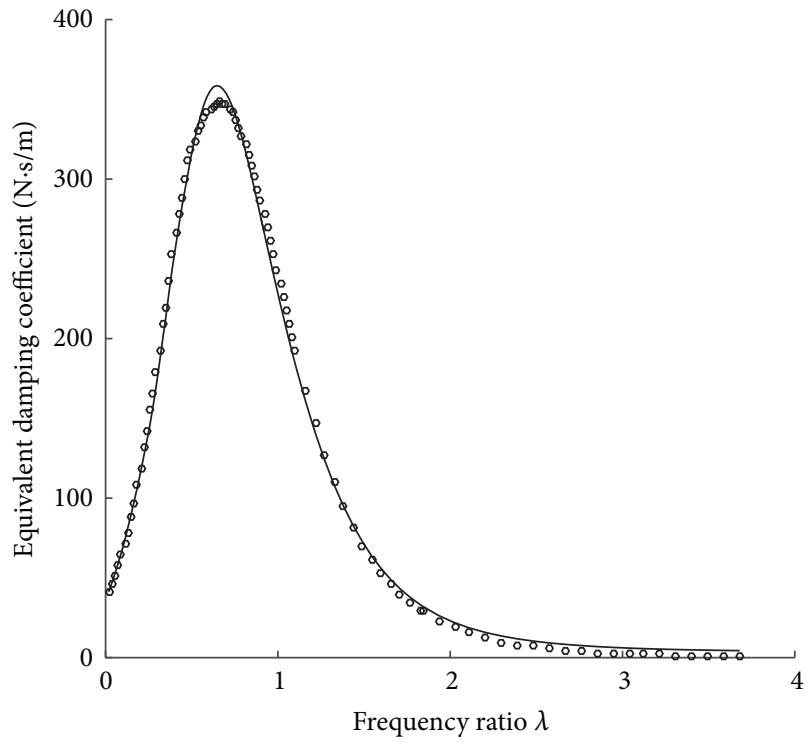

- Numerical

- Model

Figure 3: (a) Equivalent stiffness. (b) Equivalent damping coefficient.

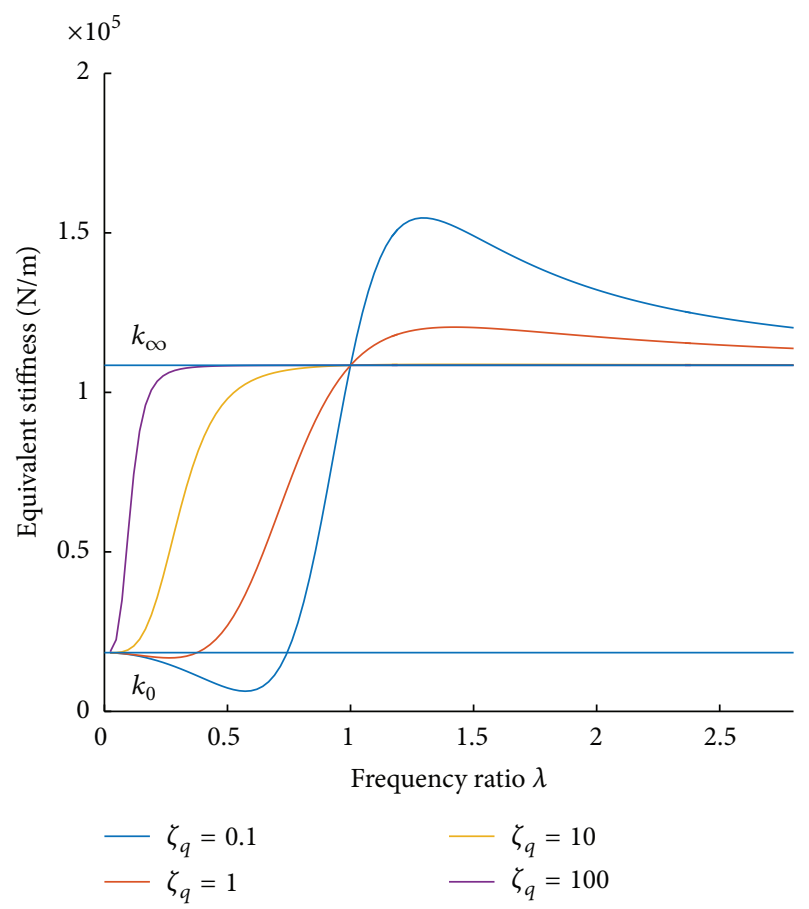

(a)

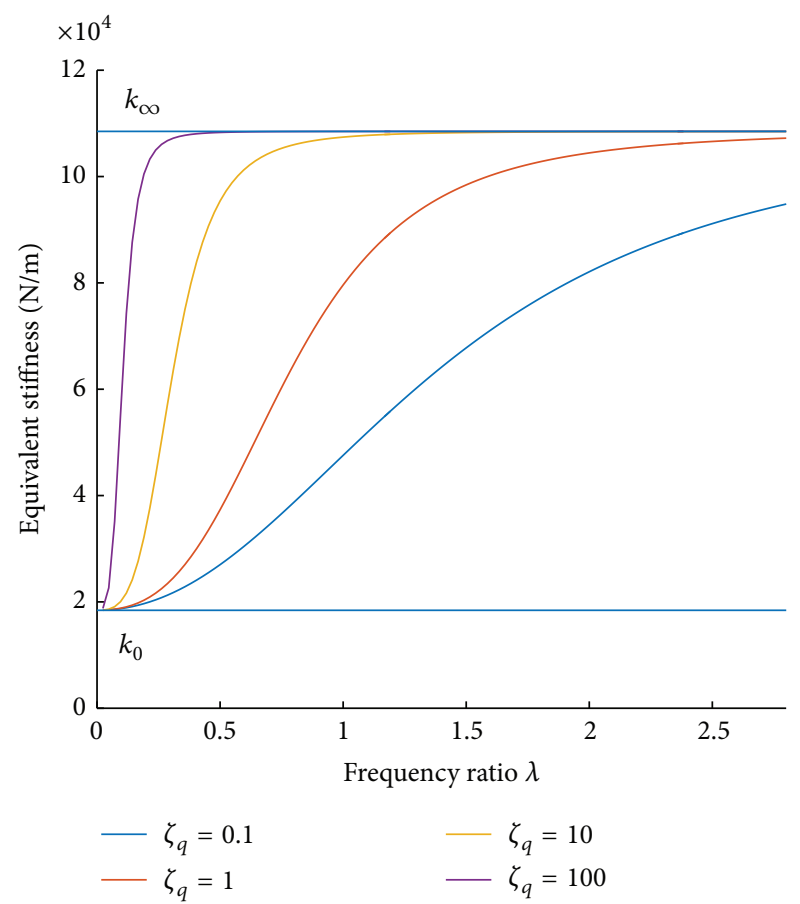

(b)

FIGURE 4: (a) Numerical simulations of the equivalent stiffness when $m_{\mathrm{eq}} \neq 0$. (b) Numerical simulations of the equivalent stiffness when $m_{\mathrm{eq}}=0$. 


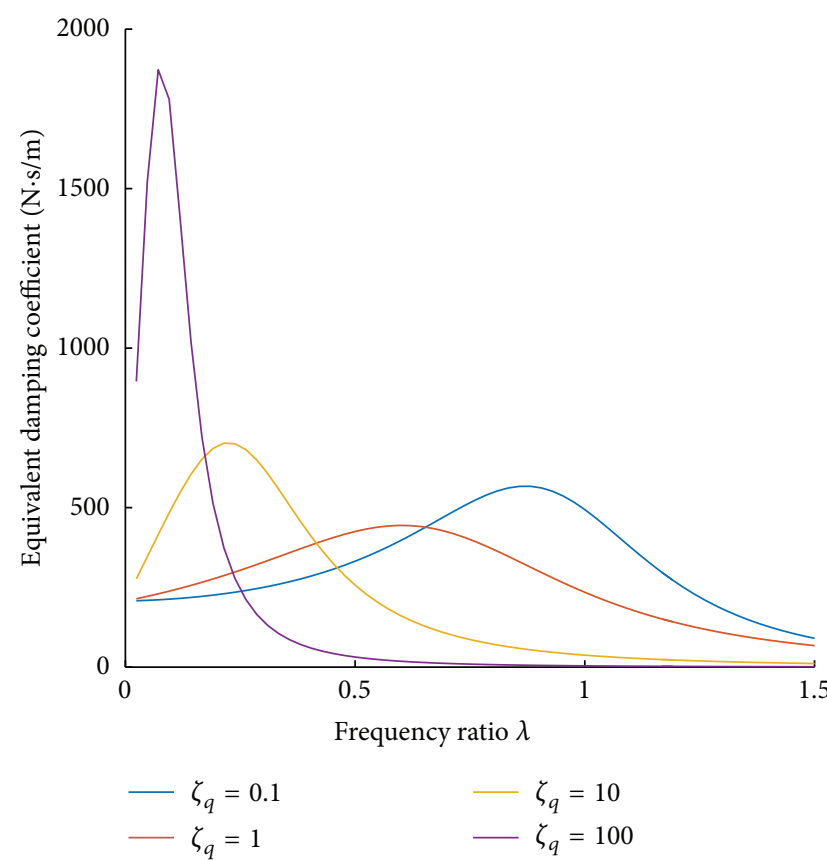

(a)

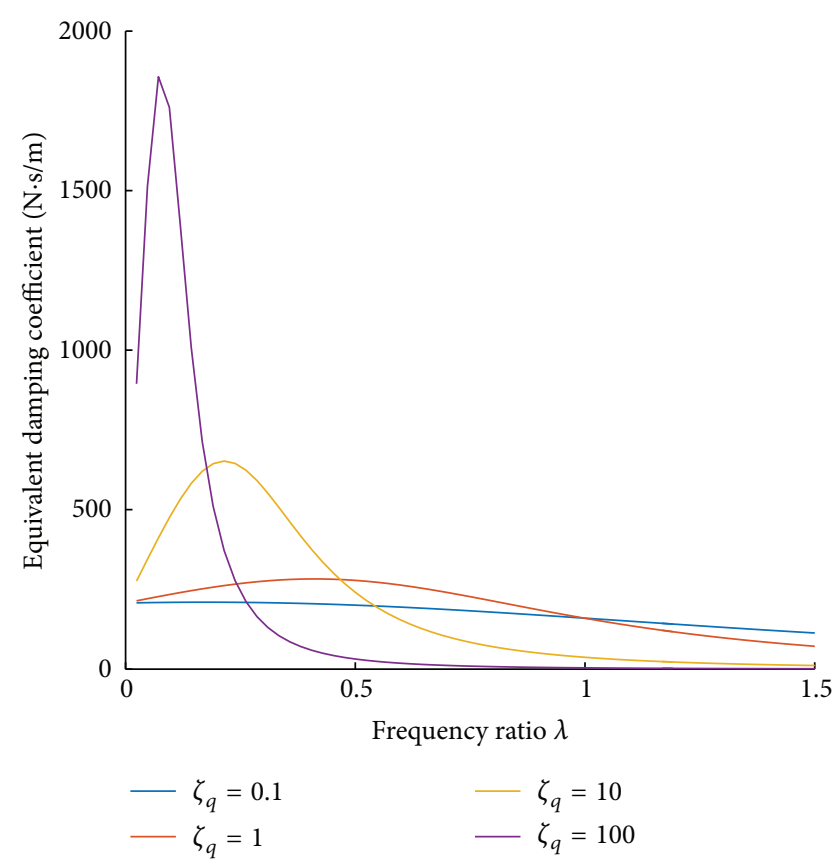

(b)

Figure 5: (a) Numerical simulations of the equivalent damping coefficient when $m_{\mathrm{eq}} \neq 0$. (b) Numerical simulations of the equivalent damping coefficient when $m_{\text {eq }}=0$.

value, such as $\zeta_{q}=100$, then it appears at the same situation as $m_{\mathrm{eq}}=0$; that is, the influence of the fluid mass in the damp channel can be neglected. This analysis indicates that $m_{\mathrm{eq}}$ can significantly influence the stiffness of the vibration isolation system at low frequency bands, thereby determining the resonance frequency of the vibration isolation system. Thus, we must choose the relevant design parameter of the isolator carefully to ensure its vibration isolation performance.

Figure 5 shows that the existence of $m_{\text {eq }}$ has two main effects on the equivalent damping coefficient. The first effect is introducing a peak point near $\lambda=1$ when the damping parameters $c_{l}$ and $c_{q}$ are relatively small, and the other is speeding up the equivalent damping decay when $\lambda>1$. When $m_{\mathrm{eq}} \neq 0$, if $\zeta_{q}$ is given a large value, then it will appear at the same situation as $m_{\mathrm{eq}}=0$; that is, the influence of the fluid mass in the damp channel can be neglected.

For a vibration isolation system, when the damping of the isolator near the resonance frequency is larger and the damping of the isolator at high frequency is smaller, the performance of the isolation system is better. Thus, introducing the fourth parameter $m_{\mathrm{eq}}$ improves the damping performance of the isolator.

The nonlinearity makes $k_{\text {eq }}$ smaller near $\omega_{v}$ and larger near $\omega_{p}$ and makes $c_{\text {eq }}$ larger near $\omega_{c}$. For a vibration isolation system, the stiffness and the damping of a vibration isolation system should be large when at a low frequency band of working frequencies. Thus, the nonlinearity of $k_{\text {eq }}$ and $\omega_{\text {eq }}$ may improve the performance of the isolator at a low frequency band when the design parameter is chosen reasonably.
TABLE 5: Key structural parameters of the sample isolator.

\begin{tabular}{lc}
\hline$d_{c}$ & $1 \mathrm{~mm}$ \\
$k_{2}$ & $100.09 \mathrm{~N} / \mathrm{mm}$ \\
$N$ & 0.181 \\
$l_{c}$ & $5 \mathrm{~mm}$ \\
\hline
\end{tabular}

\section{Experiment on the Stiffness and Damping of the Vibration Isolator}

A sample isolator was built to verify the correctness of the model. The key structural parameters of the sample isolator are shown in Table 5.

The installation status of the isolator and schematic diagram of the experiment is shown in Figure 6.

The excitation was a sinusoidal frequency sweep displacement, and the range of excitation frequency was $5-200 \mathrm{~Hz}$. The dynamic characteristics changing with the frequency of the sample isolator under different displacement excitations were tested. Figure 7 illustrates the test and numerical simulation results for comparison.

Figure 7 indicates that the numerical simulation results matched well with the experimental results; thus, the correctness of the theoretical analysis was verified. The simulated value of the sample isolator's equivalent stiffness was larger than the tested value when the excitation amplitude was relatively large. The simulated value of the equivalent damping coefficient was larger than the tested value. In general, the radial stiffness of the bellows was three to five 


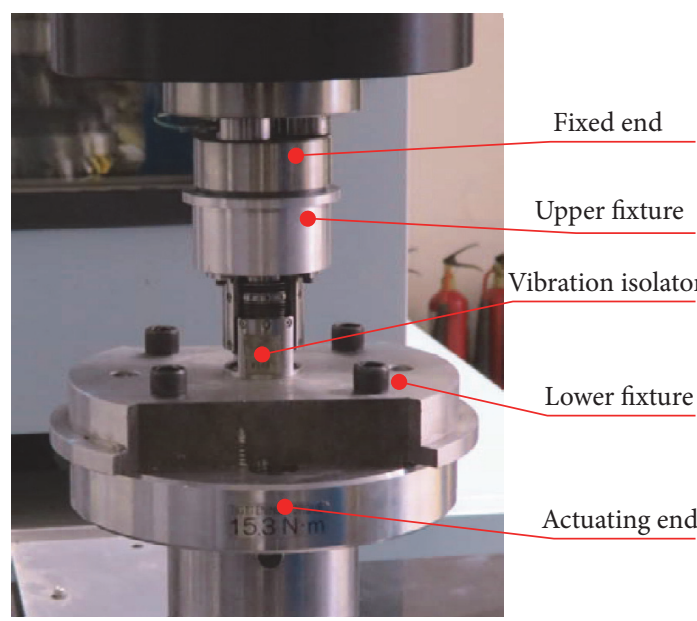

(a)

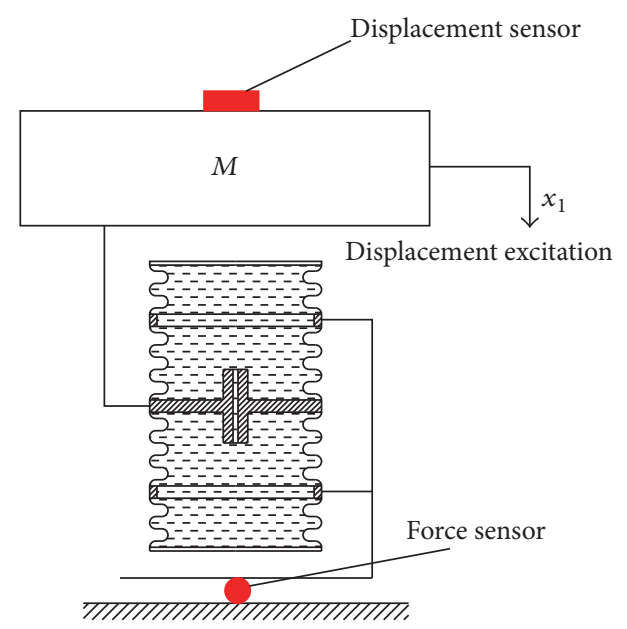

(b)

FIGURE 6: (a) Installation status of the isolator. (b) Schematic diagram of the experiment.

times larger than that of the axial stiffness. When the pressure in the cavity of the vibration isolator was large, the radial stiffness of the corrugated pipe could not be ignored. The radial stiffness of the bellows was in series with $k_{2}$, thereby resulting in the equivalent stiffness $\bar{k}_{2}<k_{2}$ and, finally, in the decrease of $k_{\infty}$. The radial stiffness of the bellows was not considered; thus, the prediction error of the dynamic stiffness was produced. Moreover, the larger the magnitude of excitation, the larger the simulated error. $T=2 \pi / \omega$ and $W$, respectively, represent the excitation period and energy dissipation within one period. $W$ can be calculated as follows:

$$
W=-\int_{0}^{T}\left(c_{1} \dot{x}_{2}+c_{q}\left|\dot{x}_{2}\right| \dot{x}_{2}\right) \dot{x}_{2} d t .
$$

The equivalent linear damping coefficient can also be defined as follows:

$$
c_{\mathrm{eq}}=-\frac{\int_{0}^{T}\left(c_{1} \dot{x}_{2}+c_{q}\left|\dot{x}_{2}\right| \dot{x}_{2}\right) \dot{x}_{2} d t}{2 \pi^{2} f X_{1}^{2}} .
$$

The combination of formulas (9) and (35) determined that when $X_{1}$ is constant, the equivalent damping coefficient $c_{\text {eq }}$ is positively correlated with $k_{2}$. Thus, when $\bar{k}_{2}<k_{2}$, the simulated value of the equivalent damping coefficient is large. According to the preceding comprehensive analysis, the experimental error is mainly caused by the incorrect input of the stiffness parameters of the bellows. This error can be corrected by improving the accuracy of the parameter value of the bellows. Therefore, the model established in this study has high accuracy when applied to the microvibration isolation system.

\section{Analysis of the Influence of the Key Parameters on the Stiffness and Damping Characteristics of the Isolator}

To isolate vibration, the natural frequency of the vibration isolation system must be lower than $\sqrt{2} / 2$ times of the frequency of the vibration disturbance. For the same vibration disturbance, the lower the natural frequency of the vibration isolation system, the better the performance of the vibration isolation; the lower the damping ratio and the higher the resonance peak value, however, the better the performance of the vibration isolation at high frequency. The stiffness and damping characteristics of a single isolator play a decisive role in the natural frequency and damping of the vibration isolation system. Thus, the influence of vibration isolator design parameters on the stiffness and damping characteristics of the vibration isolation system must be analyzed to reasonably configure the natural frequency and damping ratio of the vibration isolation system.

The design parameters that affect the stiffness and damping properties of the vibration isolator can be obtained as $l_{c}, d_{c}, k_{2}, d_{\mathrm{eq}}$, and $k_{1}$ by combining formulas (10), (11), (15), (19), and (20). Among these parameters, $k_{1}$ is parallel to the damping of the vibration isolator, which is not influenced by excitation frequency. Acting as a basic stiffness of the isolator, $k_{1}$ should meet the requirement of the supporting stiffness; hence, $k_{1}$ is not suitable for adjustment. Excitation amplitude $X_{1}$ can likewise influence the values of $\zeta_{1}$ and $\zeta_{2}$, which also affect the stiffness and damping properties of the isolator. Therefore, the analysis is made based on the effect of $X_{1}, d_{c}$, $d_{\text {eq }}, k_{2}$, and $l_{c}$ on the key points $\left(\omega_{v}, k_{v}\right),\left(\omega_{p}, k_{p}\right)\left(\omega_{c}, c_{c}\right)$.

4.1. Exciting Amplitude. According to formulas (10), (11), (15), and (25),

$$
\xi=\frac{24 \pi d_{\mathrm{eq}}^{2} \mu l_{c}+\rho d_{\mathrm{eq}}^{4} \omega X_{2}\left(\zeta_{1}+\zeta_{2}\right)}{3 d_{c}^{3} \sqrt{\pi \rho k_{2} l_{c}}} .
$$

Formula (16) shows that $X_{2}$ is positively related to $X_{1}$; thus, $\xi$ increases with the increase of $X_{1}$. The combination of formula (36) and Table 2 reveals that, with the increase of $X_{1}, \omega_{v}$ decreases while $k_{v}$ increases. When $X_{1}$ is increased to a certain extent, point $\left(\omega_{v}, k_{v}\right)$ disappears. With the increase of $X_{1}, k_{p}$ decreases while $\omega_{p}$ increases. The combination of 


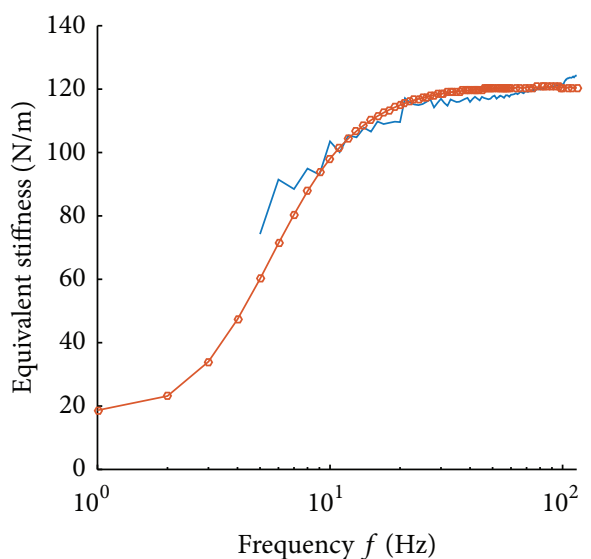

- Experiment

$\rightarrow$ Numerical

(a)

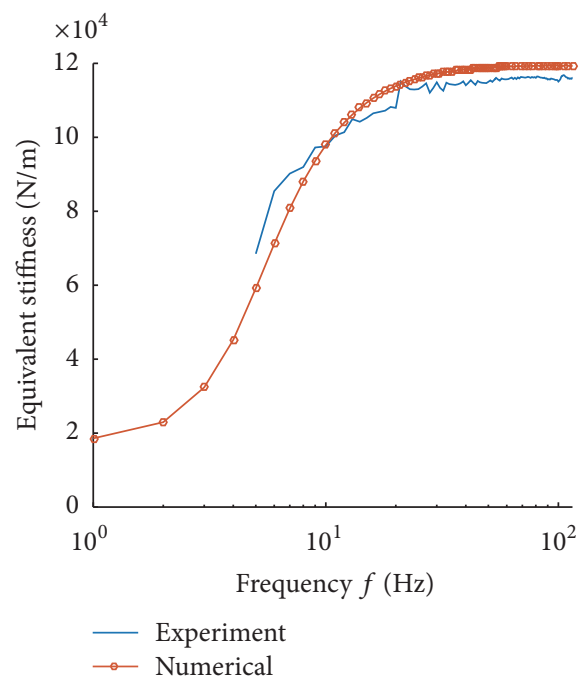

(c)

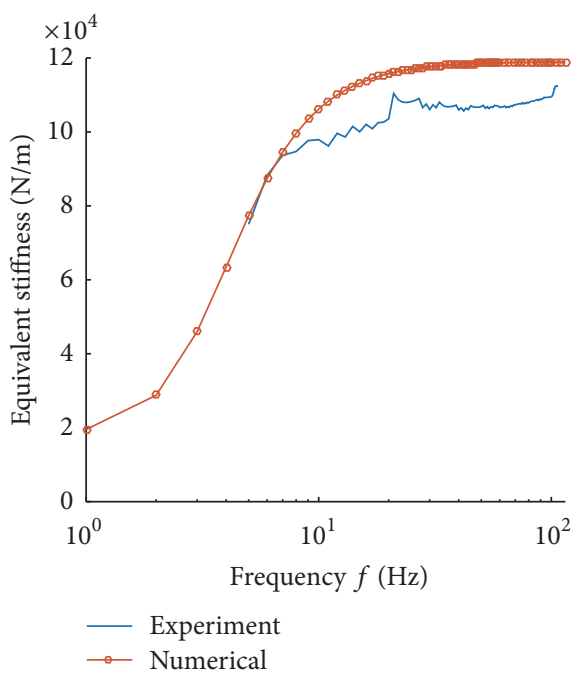

(e)

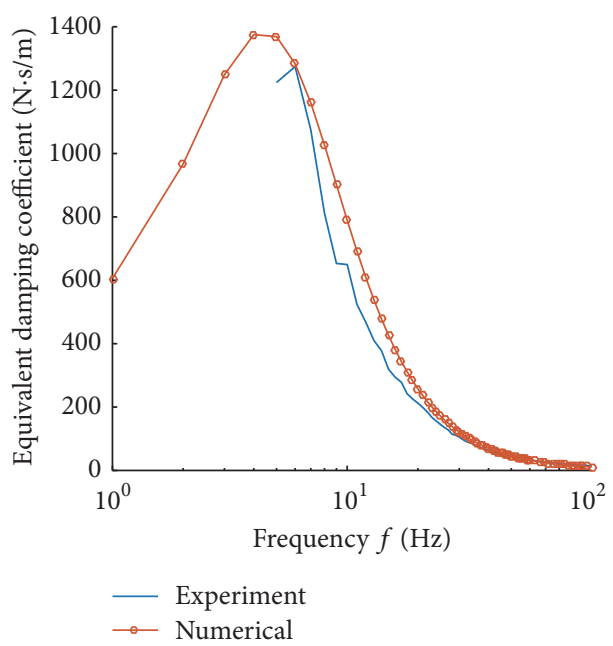

(b)

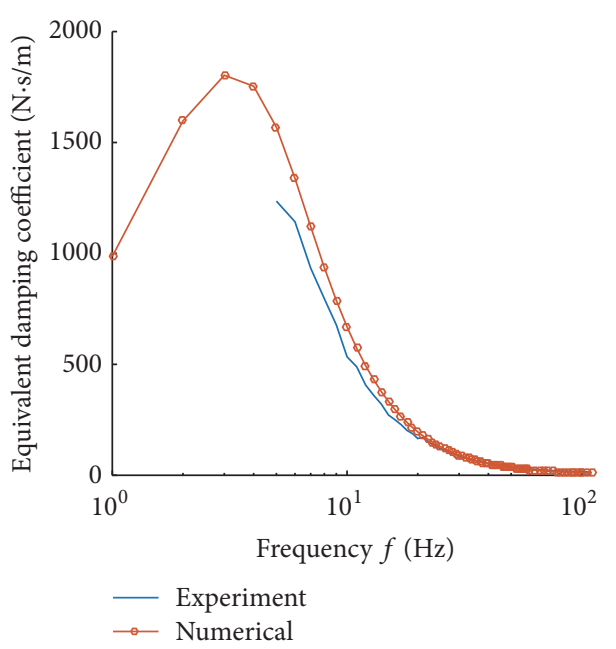

(d)

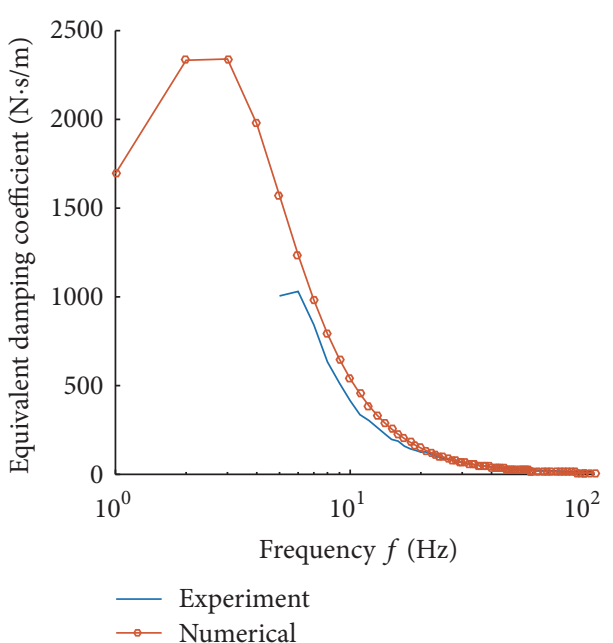

(f)

Figure 7: (a) Equivalent stiffness of 1\# isolator with an exciting amplitude of $0.2 \mathrm{~mm}$. (b) Equivalent damping coefficient of 1\# isolator with an exciting amplitude of $0.2 \mathrm{~mm}$. (c) Equivalent stiffness of 1\# isolator with an exciting amplitude of $0.4 \mathrm{~mm}$. (d) Equivalent damping coefficient of $1 \#$ isolator with an exciting amplitude of $0.4 \mathrm{~mm}$. (e) Equivalent stiffness of 1 \# isolator with an exciting amplitude of $0.8 \mathrm{~mm}$. (f) Equivalent damping coefficient of $1 \#$ isolator with an exciting amplitude of $0.8 \mathrm{~mm}$. 


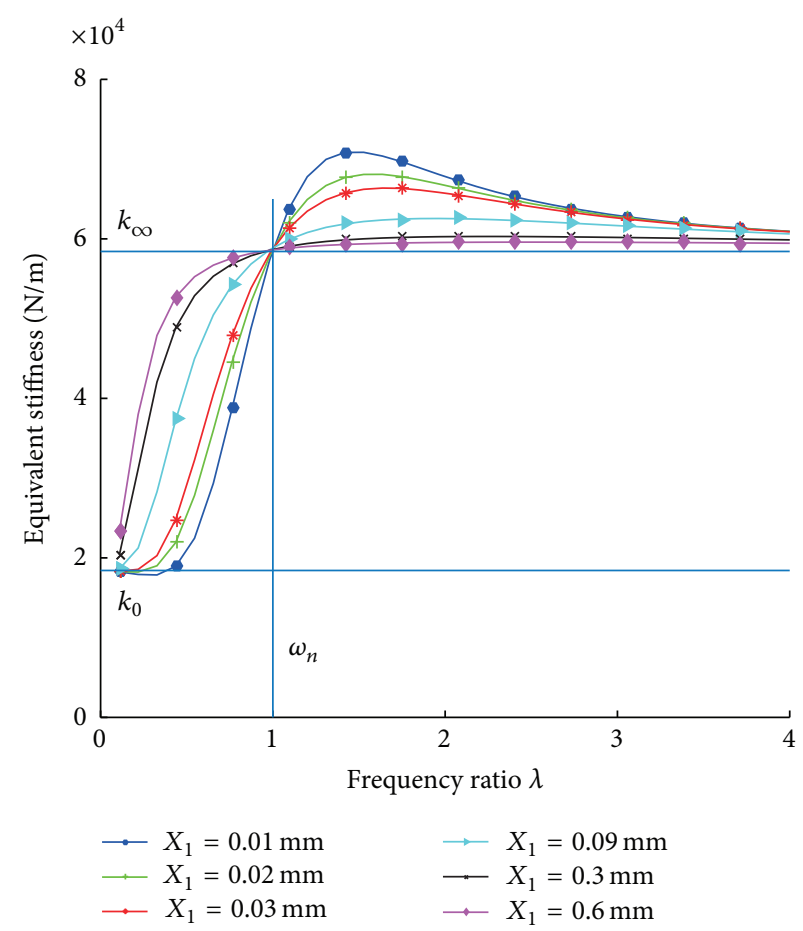

(a)

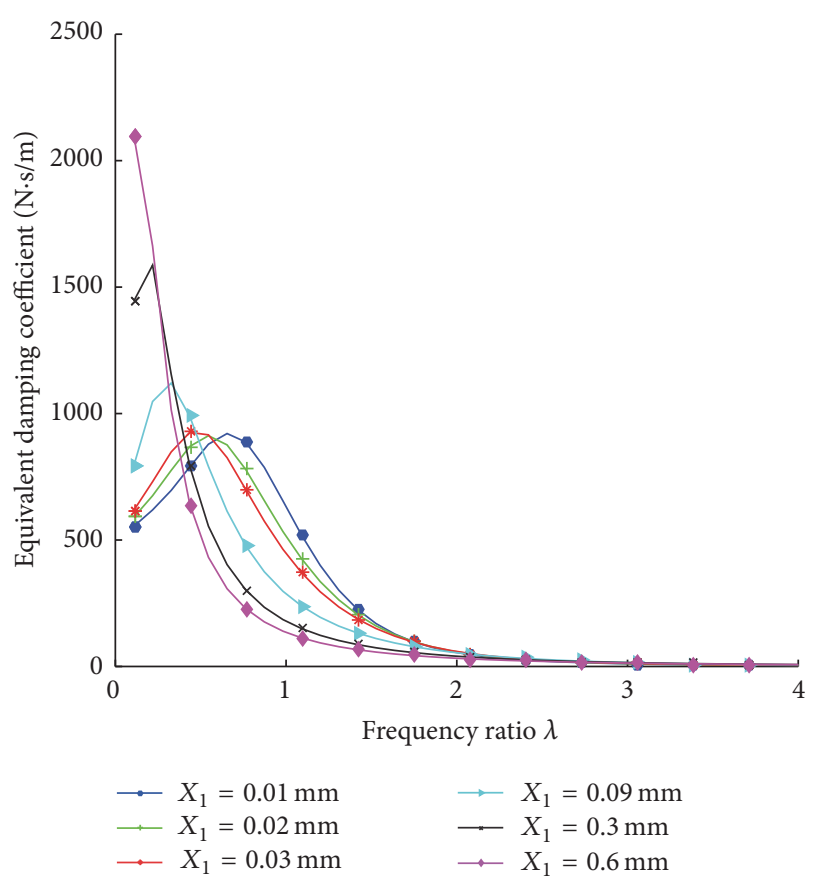

(b)

FIGURE 8: (a) Equivalent stiffness under different $X_{1}$ values. (b) Equivalent damping coefficient under different $X_{1}$ values.

formula (36) and Table 4 illustrates that $\omega_{c}$ decreases with the increase of $X_{1}$, whereas the influence of $X_{1}$ variation on $c_{c}$ is uncertain. Figure 8 shows the numerical simulation results of the equivalent stiffness and equivalent damping coefficient under different exciting amplitudes.
4.2. Damp Channel Diameter. According to formulas (24), (26), (27), (31), (32), and (36),

$$
\begin{aligned}
& \omega_{v}=\sqrt{\frac{k_{2}}{\rho l_{c}}} \sqrt{\frac{d_{c}^{2}}{d_{\mathrm{eq}}^{4}}-\frac{48 \pi \mu l_{c}+2 \rho d_{\mathrm{eq}}^{2} \omega X_{2}\left(\zeta_{1}+\zeta_{2}\right)}{3 d_{c} d_{\mathrm{eq}}^{2} \sqrt{\pi \rho k_{2} l_{c}}}} \\
& \omega_{p}=\sqrt{\frac{k_{2}}{\rho l_{c}}} \sqrt{\frac{d_{c}^{2}}{d_{\mathrm{eq}}^{4}}+\frac{48 \pi \mu l_{c}+2 \rho d_{\mathrm{eq}}^{2} \omega X_{2}\left(\zeta_{1}+\zeta_{2}\right)}{3 d_{c} d_{\mathrm{eq}}^{2} \sqrt{\pi \rho k_{2} l_{c}}}} \\
& \omega_{c}=\sqrt{\frac{k_{2}}{\rho}} \sqrt{\frac{d_{c}^{2}}{d_{e q}^{4} l_{c}}-\frac{2\left[24 \pi \mu+\rho d_{\mathrm{eq}}^{2} \omega X_{2}\left(\zeta_{1}+\zeta_{2}\right) / l_{c}\right]^{2}}{9 \pi d_{c}^{4} \rho k_{2}}} \\
& c_{c}=\frac{3 \sqrt{\pi} d_{c}^{2} k_{2} \rho l_{c}}{2\left[24 \pi \mu l_{c}+d_{\mathrm{eq}}^{2} \rho \omega X_{2}\left(\zeta_{1}+\zeta_{2}\right)\right]\left\{1-\left[24 \pi d_{\mathrm{eq}}^{2} \mu l_{c}+\rho d_{\mathrm{eq}}^{4} \omega X_{2}\left(\zeta_{1}+\zeta_{2}\right)\right]^{2} / 9 \pi d_{c}^{6} \rho k_{2} l_{c}\right\}} .
\end{aligned}
$$

Formula (37) shows that $\omega_{v}$ decreases with the increase of $d_{c}$, whereas the influence of $d_{c}$ variation on $\omega_{p}$ is uncertain. In combination with formula (36), Tables 1 and 2 show that $\xi$ decreases with the increase of $d_{c}$, thereby resulting in the increase of $k_{p}$ and in the decrease of $k_{v}$. According to the analysis in Section 2.2, when $d_{c}$ is decreased to a certain extent, point $\left(\omega_{v}, k_{v}\right)$ disappears, and $k_{p} \rightarrow k_{\infty}$. According formula (39), when $d_{c}$ increases, $\omega_{c}$ appears and then increases with the increase of $d_{c}$. According to formula (40), the influence of $d_{c}$ variation on $c_{c}$ is uncertain. Figure 9 shows the simulation results of the equivalent stiffness and equivalent damping coefficient under different damp channel 


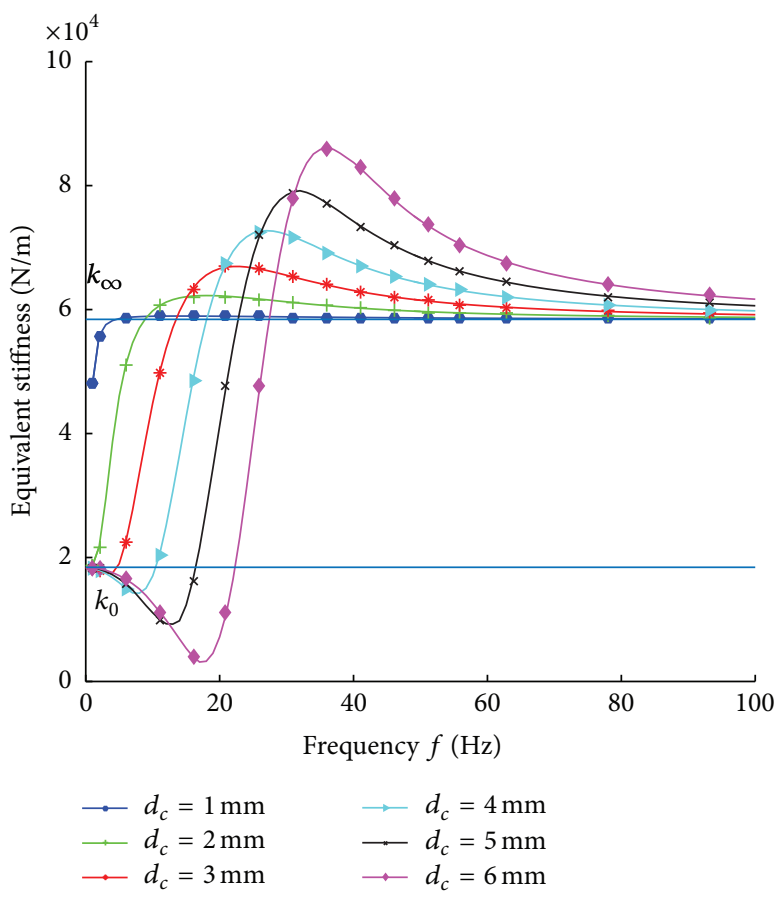

(a)

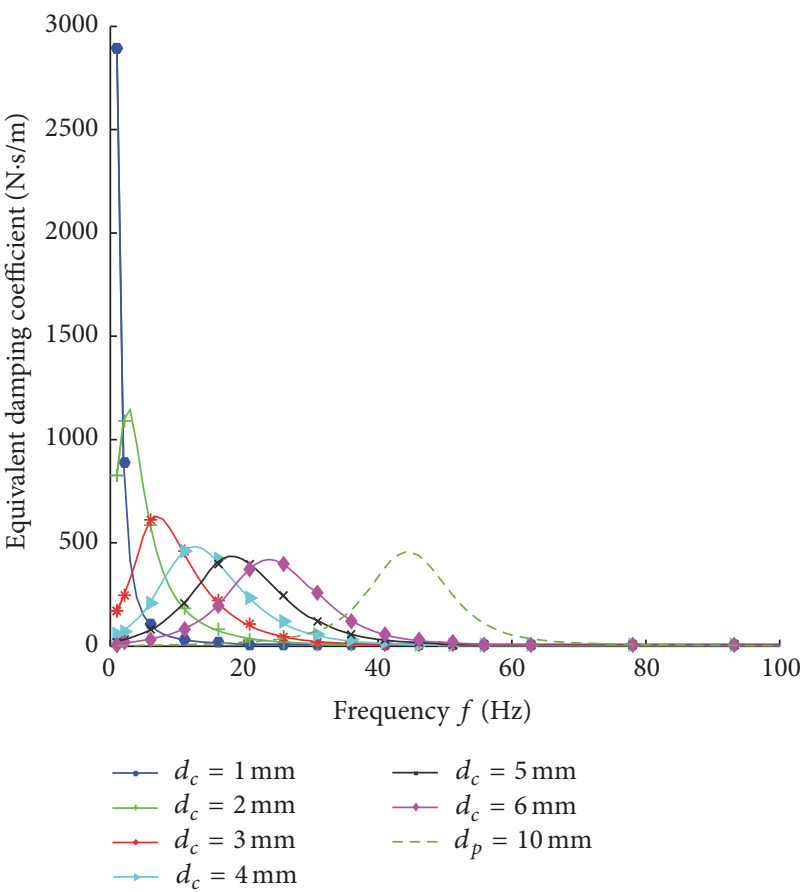

(b)

Figure 9: (a) Equivalent stiffness under different $d_{c}$ values. (b) Equivalent damping coefficient under different $d_{c}$ values.

diameter. The dotted line in Figure 9(b) represents the numerical simulation results of the equivalent damping when $d_{p}=10 \mathrm{~mm}$.

4.3. Equivalent Cylinder Diameter of the Cavities. According to formulas (37) and (38), the influence of $d_{\text {eq }}$ variation on $\omega_{v}$ is uncertain, whereas $\omega_{p}$ decreases with the increase of $d_{\text {eq }}$. In combination with formula (36), Tables 1 and 2 indicate that when $d_{\mathrm{eq}}$ increases, $\xi$ decreases, thereby resulting in the increase of $k_{v}$ and the decrease of $k_{p}$. According to the analysis in Section 2.2, when $d_{\mathrm{eq}}$ is increased to a certain extent, point $\left(\omega_{v}, k_{v}\right)$ disappears and $k_{p} \rightarrow k_{\infty}$. According to formula (39), $\omega_{c}$ decreases with the increase of $d_{\mathrm{eq}}$. When $d_{\mathrm{eq}}$ increases to a certain extent, point $\left(\omega_{c}, c_{c}\right)$ disappears. According to formula (40), the influence of $d_{\text {eq }}$ variation on $c_{c}$ is uncertain. Figure 10 shows the numerical simulation results of the equivalent stiffness and the equivalent damping coefficient under different cavity equivalent cylindrical diameters.

4.4. Sum of the Stiffness of the Bellows at the End of the Isolator. Formulas (37) and (38) show that $\omega_{v}$ and $\omega_{p}$ increase with the increase of the sum of the stiffness of the bellows at the end of the isolator $k_{2}$. According to formulas (25), (28), and (29):

$$
\begin{aligned}
& k_{v}=k_{1}+k_{2}+\frac{k_{2}^{2} m_{\mathrm{eq}}}{\bar{c}_{p}^{2}-2 \bar{c}_{p} \sqrt{k_{2} m_{\mathrm{eq}}}}, \\
& k_{p}=k_{1}+k_{2}+\frac{k_{2} m_{\mathrm{eq}}}{\bar{c}_{p}^{2} / k_{2}+2 \bar{c}_{p} \sqrt{m_{\mathrm{eq}} / k_{2}}} .
\end{aligned}
$$

The influence of $k_{2}$ variation on $k_{v}$ is uncertain, whereas $k_{p}$ increases with the increase of $k_{2}$. According to formula (39), $\omega_{c}$ increases with the increase of $k_{2}$. Formulas (22) and (25) are plugged into formula (32):

$$
c_{c}=\frac{4 k_{2} m_{\mathrm{eq}}^{2}}{4 \bar{c}_{p} m_{\mathrm{eq}}-\bar{c}_{p}^{3} / k_{2}} .
$$

$c_{c}$ increases with the increase of $k_{2}$. Figure 11 shows the numerical simulation results of the equivalent stiffness and equivalent damping coefficient under different $k_{2}$ values.

4.5. Length of Damp Channel. According to formulas (37) and (38), the influence of $l_{c}$ variation on $\omega_{v}$ and $\omega_{p}$ is uncertain. According to formulas (28), (29), and (36),

$$
\begin{aligned}
& k_{v}=k_{1}+k_{2}+\frac{9 \pi k_{2}^{2} d_{c}^{6} \rho l_{c}}{4 d_{\mathrm{eq}}^{2}\left[24 \pi \mu l_{c}+d_{\mathrm{eq}}^{2} \rho \omega X_{2}\left(\zeta_{1}+\zeta_{2}\right)\right]\left[24 d_{\mathrm{eq}}^{2} \pi \mu l_{c}-3 d_{c}^{3} \sqrt{\pi \rho k_{2} l_{c}}+d_{\mathrm{eq}}^{4} \rho \omega X_{2}\left(\zeta_{1}+\zeta_{2}\right)\right]}, \\
& k_{p}=k_{1}+k_{2}+\frac{9 \pi k_{2}^{2} d_{c}^{6} \rho l_{c}}{4 d_{\mathrm{eq}}^{2}\left[24 \pi \mu l_{c}+d_{\mathrm{eq}}^{2} \rho \omega X_{2}\left(\zeta_{1}+\zeta_{2}\right)\right]\left[24 d_{\mathrm{eq}}^{2} \pi \mu l_{c}+3 d_{c}^{3} \sqrt{\pi \rho k_{2} l_{c}}+d_{\mathrm{eq}}^{4} \rho \omega X_{2}\left(\zeta_{1}+\zeta_{2}\right)\right]} .
\end{aligned}
$$




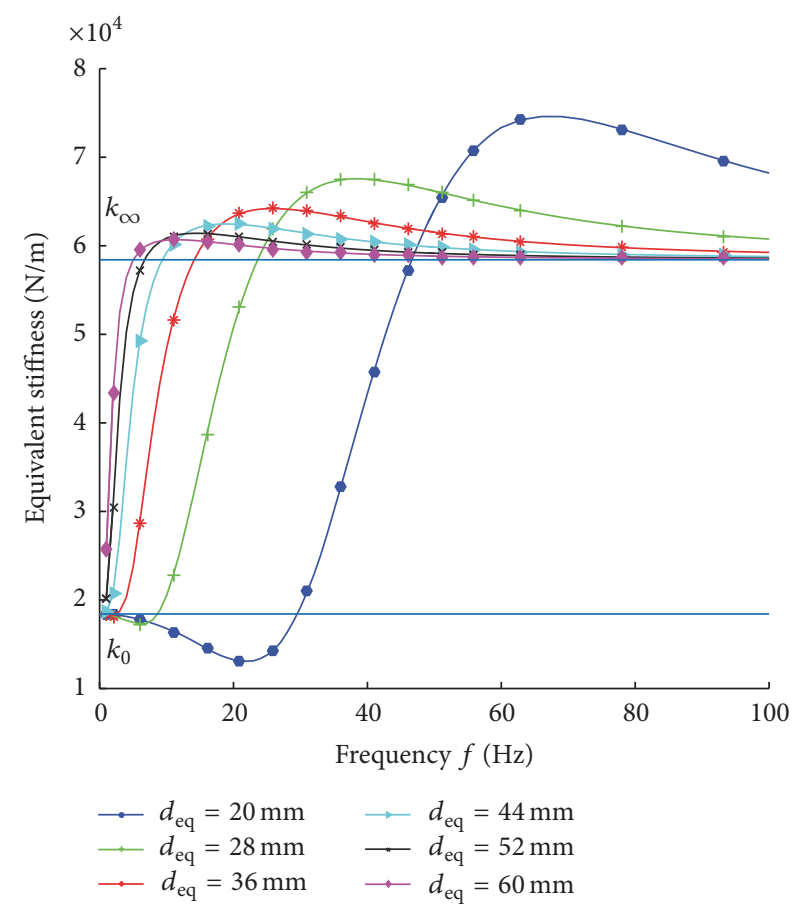

(a)

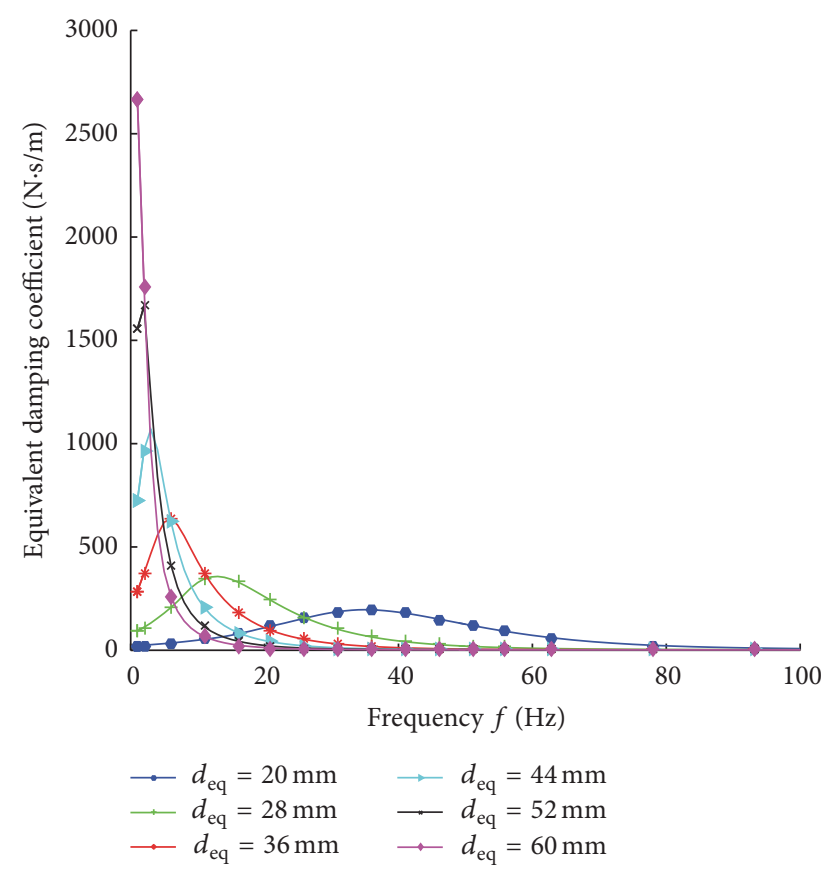

(b)

FIGURE 10: (a) Equivalent stiffness under different $d_{\mathrm{eq}}$ values. (b) Equivalent damping coefficient under different $d_{\mathrm{eq}}$ values.

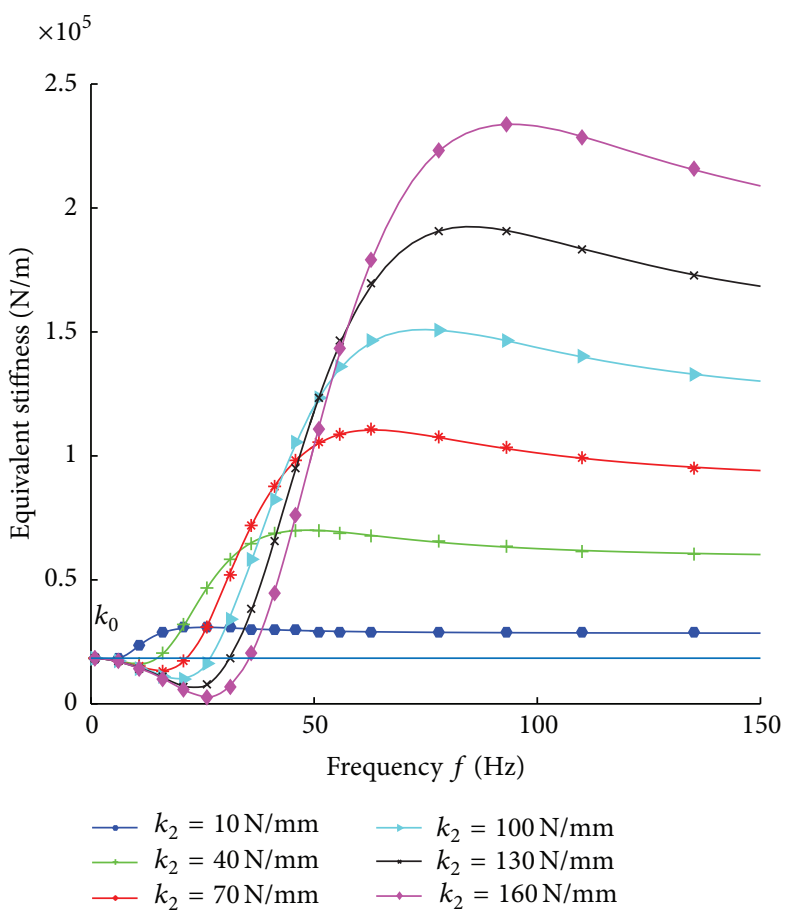

(a)

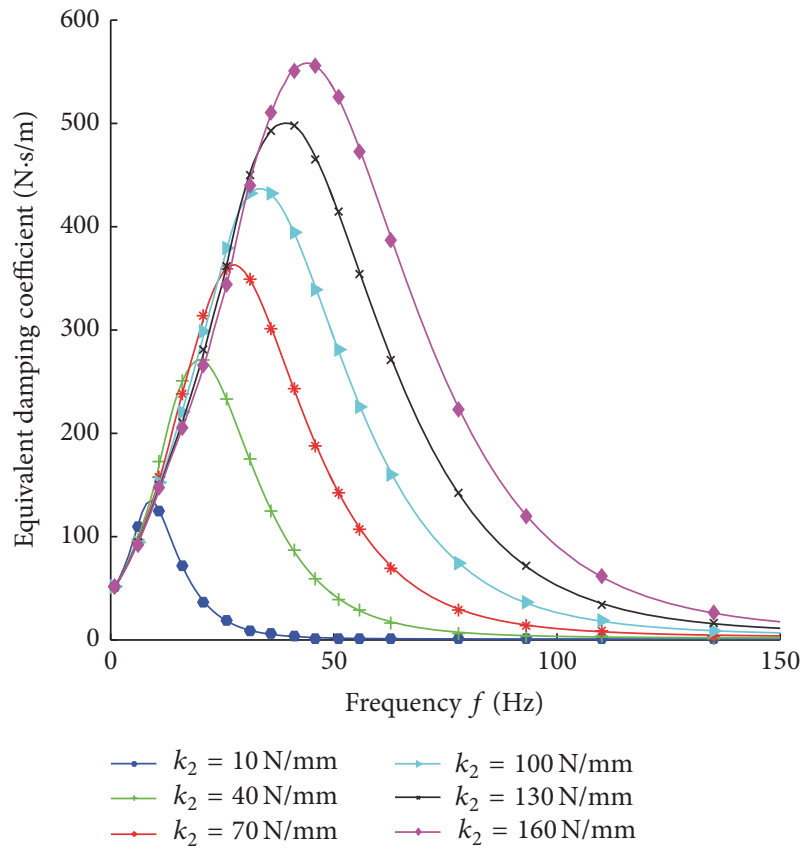

(b)

FIGURE 11: (a) Equivalent stiffness under different $k_{2}$ values. (b) Equivalent damping coefficient under different $k_{2}$ values. 


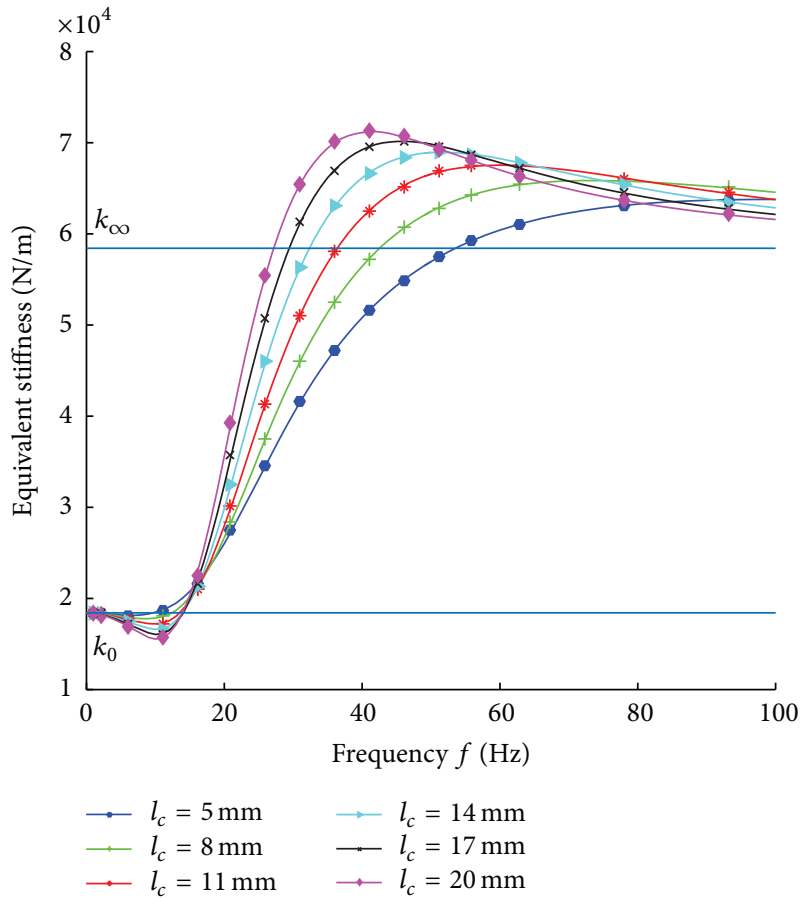

(a)

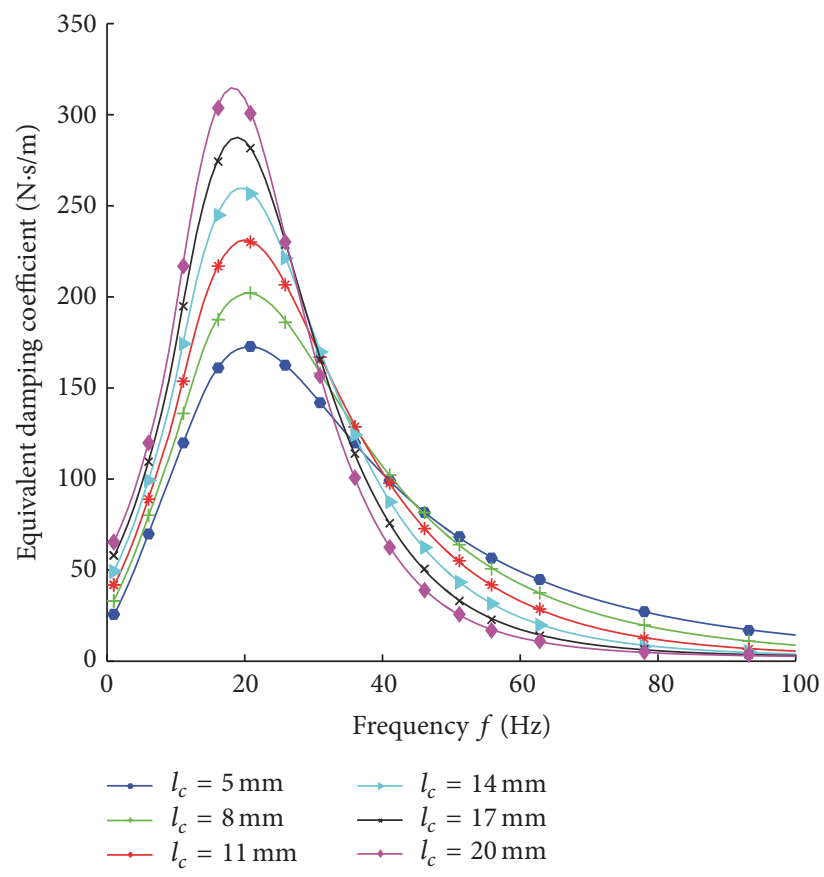

(b)

Figure 12: (a) Equivalent stiffness under different $l_{c}$ values. (b) Equivalent damping coefficient under different $l_{c}$ values.

The influence of $l_{c}$ variation on $k_{v}$ and $k_{p}$ is uncertain. According to formulas (39) and (40), the influence of $l_{c}$ variation on $\omega_{c}$ and $c_{c}$ is uncertain. Figure 12 shows the numerical simulation results of equivalent stiffness and equivalent damping coefficient under different damp channel lengths.

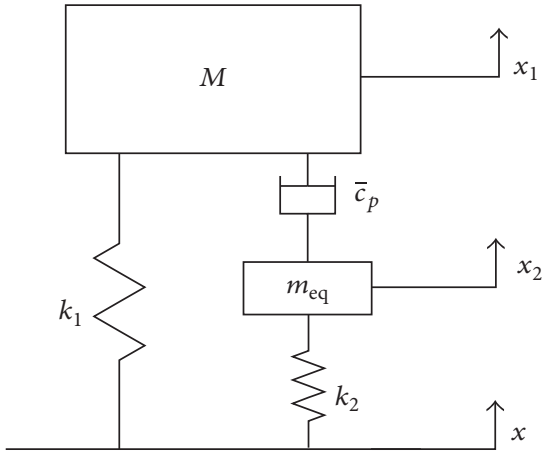

FIGURE 13: Equivalent system of the isolator with a displacement excitation base.

\section{Displacement Transmission Rate Characteristics of the Isolator}

The equivalent stiffness and equivalent damping represent the force transmission rate characteristics of the isolator. To inspect the performance of the isolator comprehensively, the displacement transmission rate characteristics of the isolator should also be studied. To study the displacement transmission rate characteristics of the isolator, the equivalent system of the isolator with a displacement excitation base is shown in Figure 13.

In Figure 13, $x$ is the displacement excitation of the base, and $x_{1}$ and $x_{2}$ are the displacement response of the load $M$ and equivalent mass of the liquid in the damp channel $m_{\text {eq, }}$, respectively.

As for the load $M$

$$
M \ddot{x}_{1}+\bar{c}_{p}\left(\dot{x}_{1}-\dot{x}_{2}\right)+k_{1}\left(x_{1}-x\right)=0 .
$$

As for the equivalent mass of the liquid in the damp channel $m_{\mathrm{eq}}$

$$
m_{\mathrm{eq}} \ddot{x}_{2}+\bar{c}_{p}\left(\dot{x}_{2}-\dot{x}_{1}\right)+k_{2}\left(x_{2}-x\right)=0 .
$$

If $x$ is the harmonic excitation, then $x, x_{1}$, and $x_{2}$ can be expressed as follows:

$$
\begin{gathered}
x=X e^{j \omega t}, \\
x_{1}=X_{1} e^{j\left(\omega t+\theta_{1}\right)}, \\
x_{2}=X_{2} e^{j\left(\omega t+\theta_{2}\right)} .
\end{gathered}
$$

Formula (48) is plugged into formulas (46) and (47), and the following formula can be obtained:

$$
\begin{aligned}
x_{1}\left(-\omega^{2} M+j \omega \bar{c}_{p}+k_{1}\right) & =x_{2} j \omega \bar{c}_{p}+k_{1} x, \\
x_{2}\left(-\omega^{2} m_{\mathrm{eq}}+j \omega \bar{c}_{p}+k_{2}\right) & =x_{1} j \omega \bar{c}_{p}+k_{2} x .
\end{aligned}
$$

Assuming that $A_{1}=-\omega^{2} M+j \omega \bar{c}_{p}+k_{1}, A_{2}=-\omega^{2} m_{\mathrm{eq}}+$ $j \omega \bar{c}_{p}+k_{2}$ and $A_{3}=j \omega \bar{c}_{p}$, From formula (49), we can obtain

$$
\frac{x_{1}}{x}=\frac{A_{3} k_{2}+A_{2} k_{1}}{A_{1} A_{2}-A_{3}^{2}} \text {. }
$$




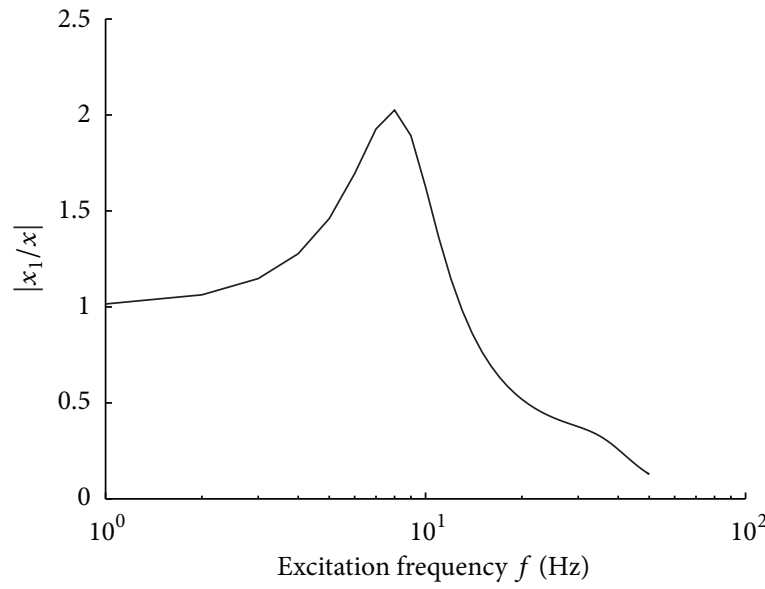

(a)

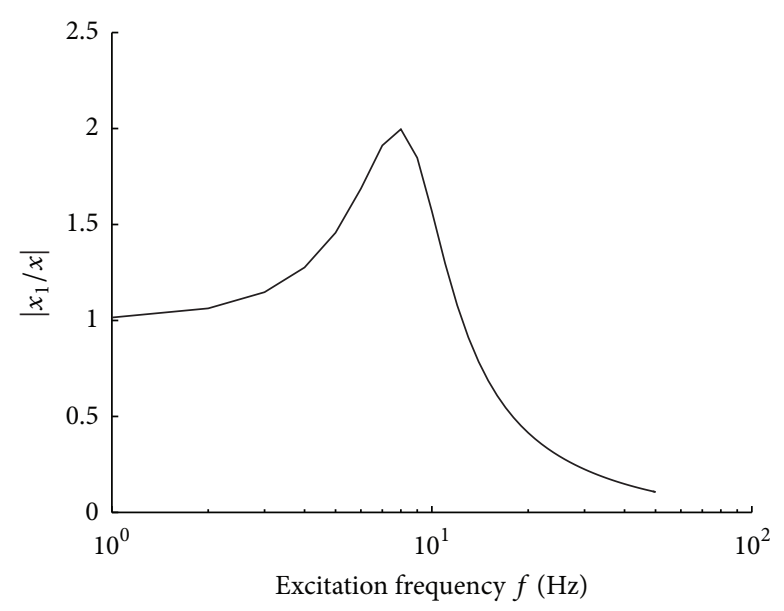

(b)

FIGURE 14: (a) Amplitude-frequency characteristic when $m_{\mathrm{eq}} \neq 0$. (b) Amplitude-frequency characteristic when $m_{\mathrm{eq}}=0$.

By plugging $A_{1}, A_{2}$, and $A_{3}$ into formula (50), we can obtain the displacement transfer function

$$
\begin{aligned}
T_{1} & =\frac{x_{1}}{x} \\
& =\frac{k_{1} k_{2}+j \omega \bar{c}_{p}\left(k_{1}+k_{2}\right)-k_{1} m_{\mathrm{eq}} \omega^{2}}{\bar{c}_{p}^{2} \omega^{2}+\left(k_{2}+j \omega \bar{c}_{p}-m_{\mathrm{eq}} \omega^{2}\right)\left(k_{1}+j \omega \bar{c}_{p}-M \omega^{2}\right)} .
\end{aligned}
$$

For comparison, assuming that $m_{\mathrm{eq}}=0$, then the displacement transfer function is

$$
\begin{aligned}
T_{2} & =\frac{x_{1}}{x} \\
& =\frac{k_{1} k_{2}+j \omega \bar{c}_{p}\left(k_{1}+k_{2}\right)}{k_{1} k_{2}+j \omega \bar{c}_{p}\left(k_{1}+k_{2}\right)-k_{2} M \omega^{2}-j \bar{c}_{p} M \omega^{3}} .
\end{aligned}
$$

Thus, we can obtain the amplitude-frequency characteristic of the displacement transfer function $\left|x_{1} / x\right|$ when $m_{\mathrm{eq}} \neq 0$ and $m_{\mathrm{eq}}=0$, and it is shown in Figures 14(a) and 14(b), respectively.

From Figure 14, we can see that the existence of $m_{\text {eq }}$ gives the amplitude-frequency characteristic of the displacement transfer function curve an additional peak at a high frequency band, thereby resulting in the displacement transfer function of the novel isolator decaying twice at high frequency band. By contrast, the displacement transfer function of the novel isolator decays only once at a high frequency band when $m_{\text {eq }}=0$. The existence of $m_{\text {eq }}$ improves the performance of the isolator at a high frequency band. Moreover, the relevant parameter should be chosen carefully to ensure a reasonable frequency of the second curve peak, thereby preventing the performance of the isolator from being influenced at the frequency of the second peak.

\section{Conclusion}

This study conducted a theoretical analysis, experimental verification, and analysis of the influence on the dynamic characteristics of the key parameter variation of the new type of fluid damping vibration isolator with bellows. The following conclusions were drawn from the results.

(1) The model established in this study has high accuracy when applied to microvibration isolation.

(2) The fourth parameter $m_{\text {eq }}$ improves the performance of the isolator at a high frequency band.

(3) The parameters of the vibration isolator can be identified according to the fixed points $\left(0, k_{1}\right),\left(\omega_{n}, k_{1}+\right.$ $\left.k_{2}\right)$, and $\left(\infty, k_{1}+k_{2}\right)$ of the equivalent stiffness curve of the vibration isolator with different excitation amplitudes.

(4) According to the influence of the key parameter variation on the performance of the vibration isolator, the rule of the influence of key design parameters of the vibration isolator on the equivalent stiffness and damping coefficient of the isolator is as follows:

$\begin{array}{ccccccc} & \omega_{v} & k_{v} & \omega_{p} & k_{p} & \omega_{c} & c_{c} \\ X_{1} & \downarrow & \uparrow & \uparrow & \downarrow & \downarrow & \text { uncertain } \\ d_{c} & \uparrow & \downarrow & \text { uncertain } & \uparrow & \uparrow & \text { uncertain } \\ d_{\mathrm{eq}} & \text { uncertain } & \uparrow & \downarrow & \uparrow & \downarrow & \text { uncertain } \\ k_{2} & \uparrow & \text { uncertain } & \uparrow & \uparrow & \uparrow & \uparrow \\ l_{c} & \text { uncertain } & \text { uncertain } & \text { uncertain } & \text { uncertain } & \text { uncertain } & \text { uncertain }\end{array}$


(5) When the vibration isolator is designed for a vibration isolation system, $k_{1}$ can initially be determined according to the static stiffness demand of the isolation system, whereas $k_{2}$ can be determined by the stiffness demand at high frequency. Then, according to the rule of $d_{c}, l_{c}$, and $d_{\mathrm{eq}}$ influencing the stiffness and damping of the isolator and the performance demand of the vibration isolation system, $d_{c}, l_{c}$, and $d_{\text {eq }}$ of each vibration isolator can be designed. Thus, the design of the isolators can be accomplished.

\section{Competing Interests}

The authors declare that there is no conflict of interests regarding the publication of this paper.

\section{Acknowledgments}

The authors would like to thank the College of Automotive Engineering, Jilin University, Changchun, Jilin, China, for their support in this project.

\section{References}

[1] R. Ullio and F. Marta, SM98-109/433 Artemis Micro-Vibration Environment Prediction, vol. 428, 1999.

[2] N. Jedrich, D. Zimbelman, M. Turczyn et al., "Cryo cooler induced micro-vibration disturbances to the hubble space telescope," NASA STI-RECON Technical Report 2, NASA, 2002.

[3] T. Wacker, L. Weimer, and K. Eckert, "GOCE platform microvibration verification by test and analysis," in Proceedings of the European Conference on Spacecraft Structures, Materials and Mechanical Testing (ESA SP-581 '05), Noordwijk, The Netherlands, May 2005.

[4] D.-K. Kim, "Micro-vibration model and parameter estimation method of a reaction wheel assembly," Journal of Sound and Vibration, vol. 333, no. 18, pp. 4214-4231, 2014.

[5] Z. Zhang, G. S. Aglietti, and W. Zhou, "Microvibrations induced by a cantilevered wheel assembly with a soft-suspension system," AIAA Journal, vol. 49, no. 5, pp. 1067-1079, 2011.

[6] C. Liu, X. Jing, S. Daley, and F. Li, "Recent advances in microvibration isolation," Mechanical Systems and Signal Processing, vol. 56, no. 1, pp. 55-80, 2015.

[7] J. B. Breckinridge, "Use of a passive reaction wheel jitter isolation system to meet the Advanced X-Ray astrophysics facility imaging performance requirements," in Space Telescopes and Instruments V, vol. 3356 of Proceedings of SPIE, pp. 1078-1094, The International Society for Optical Engineering, 1998.

[8] A. J. Bronowicki, "Vibration isolator for large space telescopes," Journal of Spacecraft and Rockets, vol. 43, no. 1, pp. 45-53, 2006.

[9] E. H. Anderson, J. P. Fumo, and R. S. Erwin, "Satellite ultraquiet isolation technology experiment (SUITE)," in Proceedings of the 2000 IEEE Aerospace Conference, pp. 299-313, Big Sky, Mont, USA, March 2000.

[10] D. Cunningham and P. Davis, "A multiaxis passive isolation system for a magnetic bearing reaction wheel," Advances in the Astronautical Sciences, vol. 95, p. 454, 1993.

[11] E. I. Rivin, "Passive vibration isolation," Applied Mechanics Reviews, vol. 57, no. 6, pp. B31-B32, 2003.
[12] P. Davis, D. Cunningham, and J. Harrell, "Advanced $1.5 \mathrm{~Hz}$ passive viscous isolation system," in Proceedings of the 35th AIAA/ASME/ASCE/AHS/ASC Structures, Structural Dynamics, and Materials Conference, pp. 2655-2665, April 1994.

[13] D. Kamesh, R. Pandiyan, and A. Ghosal, "Passive vibration isolation of reaction wheel disturbances using a low frequency flexible space platform," Journal of Sound \& Vibration, vol. 331, no. 6, pp. 1310-1330, 2012.

[14] R. A. Ibrahim, "Recent advances in nonlinear passive vibration isolators," Journal of Sound and Vibration, vol. 314, no. 3-5, pp. 371-452, 2008.

[15] J. Sun, X. Huang, X. Liu, F. Xiao, and H. Hua, "Study on the force transmissibility of vibration isolators with geometric nonlinear damping," Nonlinear Dynamics, vol. 74, no. 4, pp. 1103-1112, 2013.

[16] P. F. Guo, Z. Q. Lang, and Z. K. Peng, "Analysis and design of the force and displacement transmissibility of nonlinear viscous damper based vibration isolation systems," Nonlinear Dynamics, vol. 67, no. 4, pp. 2671-2687, 2012.

[17] Q. Lv and Z. Yao, "Analysis of the effects of nonlinear viscous damping on vibration isolator," Nonlinear Dynamics, vol. 79, no. 4, pp. 2325-2332, 2015.

[18] Q. J. Yang and L. Liao, "Research of isolator using bellow and fluid damping," Chinese Hydraulics \& Pneumatics, 2006.

[19] M. J. Brennan, A. Carrella, T. P. Waters, and V. Lopes Jr., "On the dynamic behaviour of a mass supported by a parallel combination of a spring and an elastically connected damper," Journal of Sound and Vibration, vol. 309, no. 3-5, pp. 823-837, 2008.

[20] X. J. Jing and Z. Q. Lang, "Frequency domain analysis of a dimensionless cubic nonlinear damping system subject to harmonic input," Nonlinear Dynamics, vol. 58, no. 3, pp. 469-485, 2009.

[21] Q. J. Yang and L. Liao, Research of Isolator Using Bellow and Fluid Damping, Chinese Hydraulics \& Pneumatics, 2006.

[22] M. Hamada, S. Takezono, K. Okamoto et al., "Strength of Ushaped bellows: 1st report, case of axial loading," Transactions of the Japan Society of Mechanical Engineers, vol. 31, no. 223, pp. 389-396, 1965.

[23] R. Fan and Z. Lu, "Fixed points on the nonlinear dynamic properties of hydraulic engine mounts and parameter identification method: experiment and theory," Journal of Sound and Vibration, vol. 305, no. 4-5, pp. 703-727, 2007. 


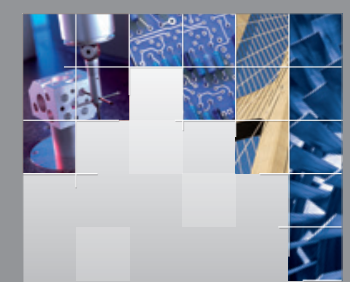

\section{Enfincering}
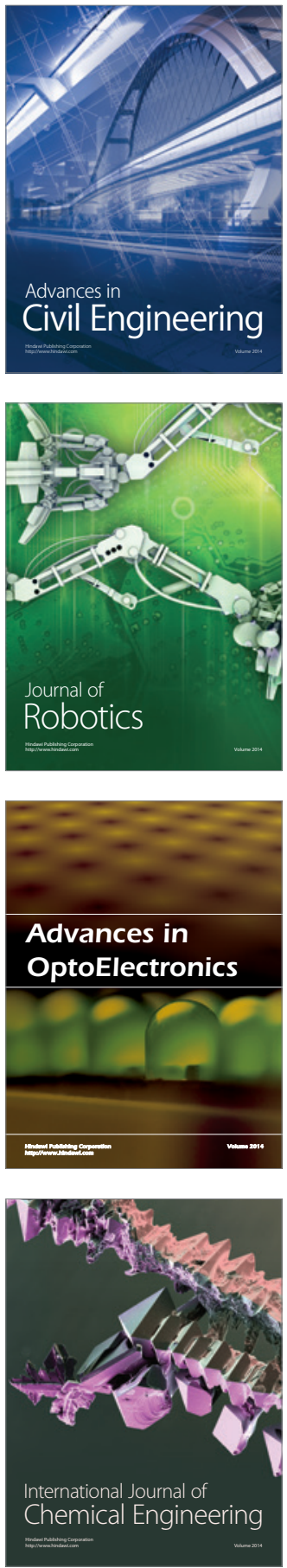

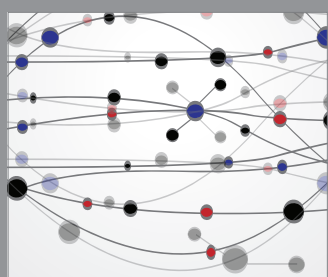

The Scientific World Journal

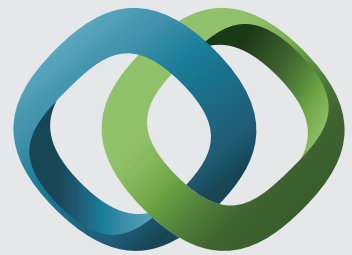

\section{Hindawi}

Submit your manuscripts at

http://www.hindawi.com
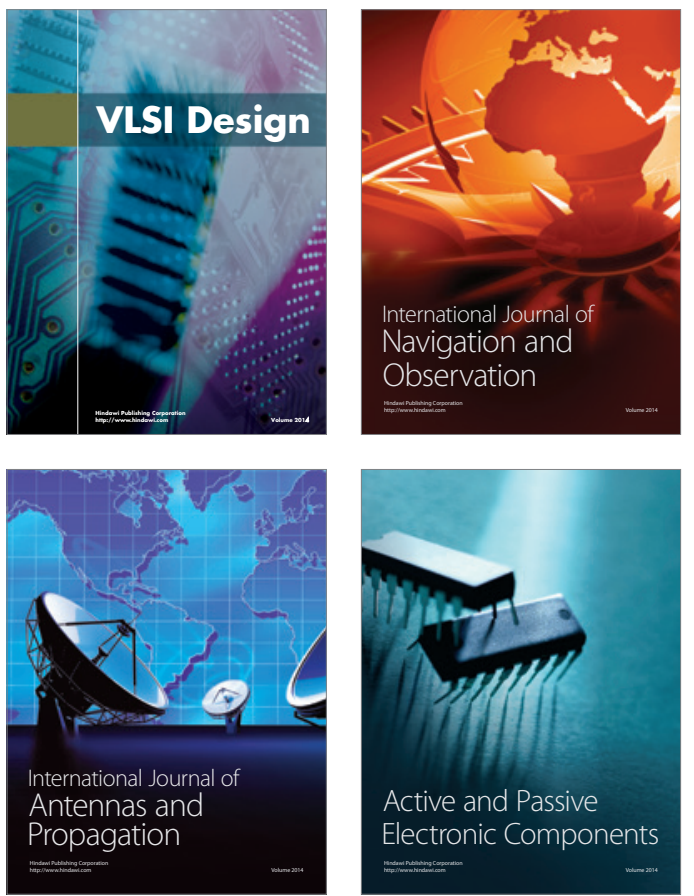
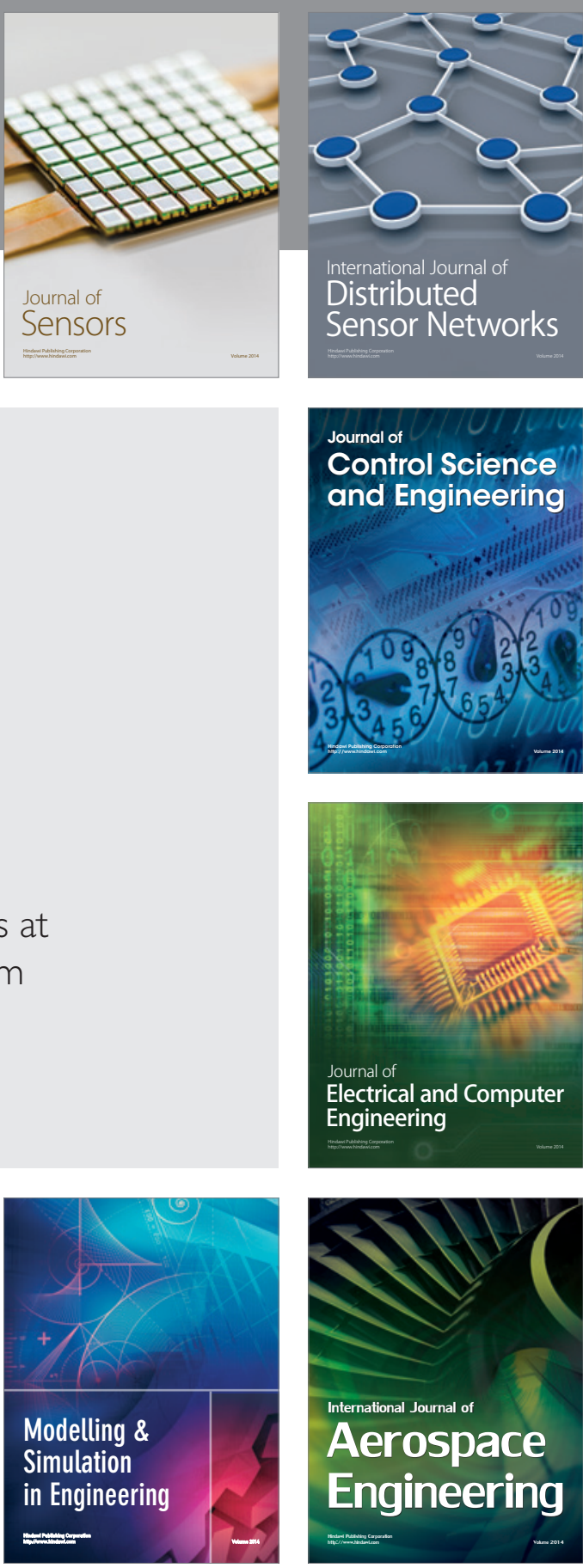

International Journal of

Distributed

Sensor Networks

Journal of

Control Science

and Engineering
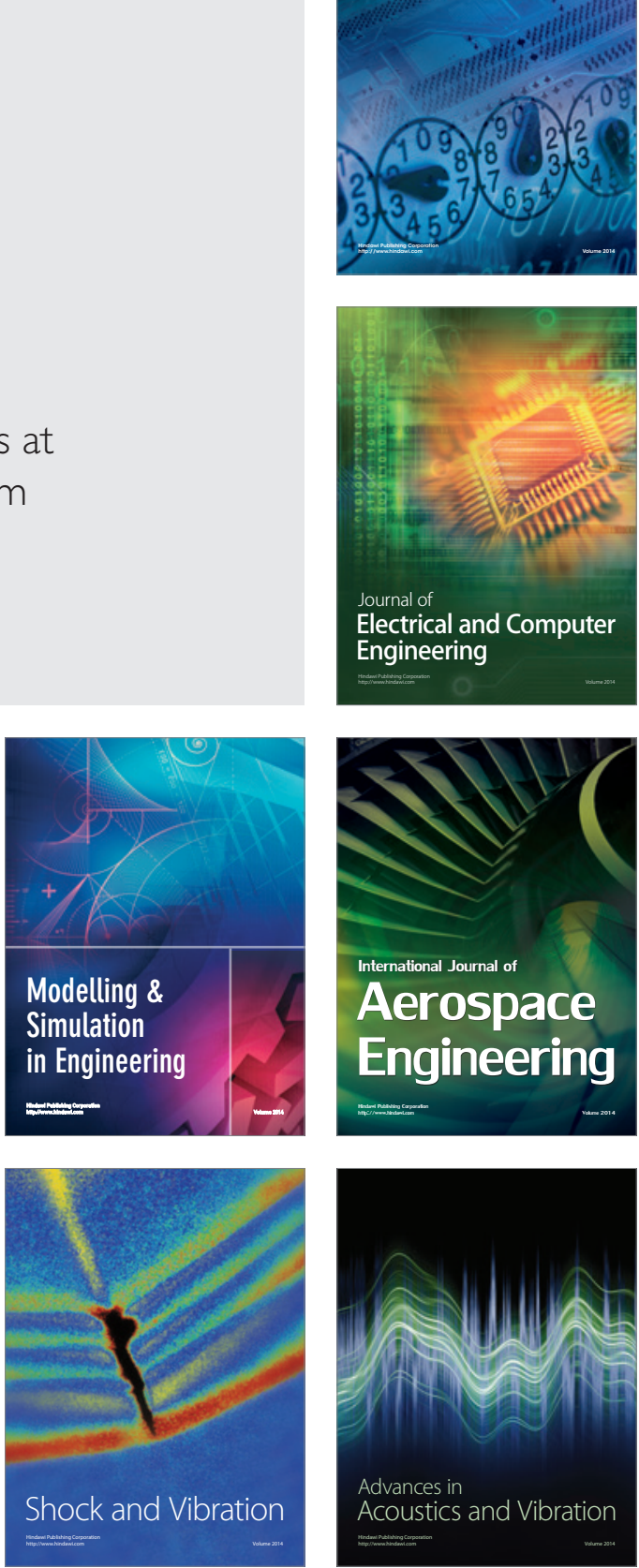